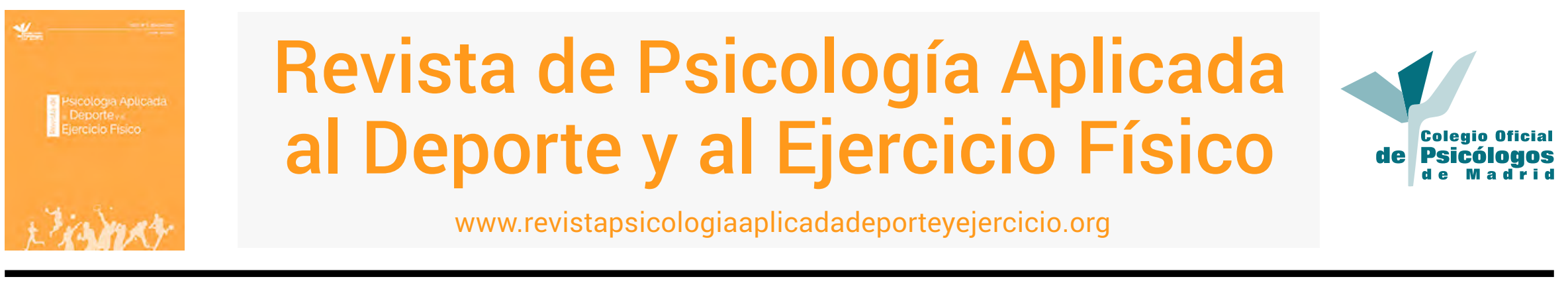

\title{
Adicción a correr: una revisión desde sus inicios hasta la actualidad
}

\author{
Abel Nogueira López, Alfonso Salguero del Valle y Sara Márquez Rosa \\ Universidad de León, España
}

RESUMEN: El fenómeno del running ha sido y continúa siendo uno de los deportes que más adeptos ha conseguido captar en los últimos años, ya que son muchas las personas que de manera regular salen a la calle día tras día a correr. Esta práctica deportiva se ha convertido en un comportamiento habitual en nuestra sociedad al ser considerado como una de las mejores actividades para mantener y mejorar la salud física, mental y psicosocial. El problema surge cuando correr se convierte en una necesidad perjudicial, provocando consecuencias negativas para la salud y dando lugar a lo que se conoce como Adicción al Ejercicio - "adicción positiva (APC); actividad agradable que genera un placer extremo" y "adicción negativa (ANC); cuando la vida de una persona se rige por esta actividad"-. Ante esta situación, el objetivo de este trabajo es conocer el estado actual de las investigaciones sobre adicción al ejercicio, centrándonos en el "running". La búsqueda de artículos se realizó en las bases de datos Pubmed, Scopus, Web of Science y EBSCOhost utilizando combinaciones de las palabras clave: "deporte", "running", "correr", "adicción”, "dependencia”, "marathon”. Los principales resultados indican que la adicción al deporte podría incluirse dentro de la categoría de los trastornos de la conducta, ya que cumple con la gran mayoría de los criterios diagnósticos y sobre todo por las graves consecuencias que esta puede ocasionar en el día a día de quienes la sufren. Por último, proponemos unas recomendaciones aplicadas que según nuestro criterio pueden tenerse en cuenta como soluciones y alternativas para aumentar el número de personas que se aprovechan de todos los beneficios de la práctica deportiva regular.

PALABRAS CLAVES: Running, adicción, deporte, salud, revisión.

\section{Addiction to running: a review from its beginnings to the present day}

ABSTRACT: Running has been and continues to be one of the sports which have attracted more recruits in recent years. Thousands of people run every day in the streets, parks, etc. Running has become a usual behavior in our society, as it is considered one of the best activities to maintain and improve physical, psychological, and psychosocial health. The problem appears when running becomes a harmful need and has negative consequences for health, leading to the so-called exercise addiction - "positive addiction (PAR); an enjoyable activity which causes an extreme pleasure", and "negative addiction (NAR), which occurs when the activity controls the life of a person". The main objective of this study was to ascertain the current state of research on exercise addiction, with a special focus on running. A literature search suggested that we are facing a phenomenon that meets most of the criteria to be considered as a behavioral disorder. However, additional research is needed to be able to better understand and, above all, to continue to answer the multiple questions related to its manifestation. The literature search was carried out in the Pubmed, Scopus, Web of Science and EBSCOhost databases using combinations of the keywords "sport"; "running"; "running addiction"; "dependence", and "marathon". The main results show that exercise addiction may be included in the category of behavioral disorders, because it fulfills the majority of diagnostic criteria and, above all, because of its serious consequences for the daily life of those who suffer it. Finally, some recommendations are proposed which, in our opinion, may be considered as solutions and alternatives to increase the number of people who take advantage of all the benefits of regular sport practice.

KEYWORDS: Running, addiction, sport, healthy, review.

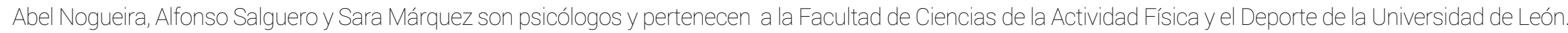

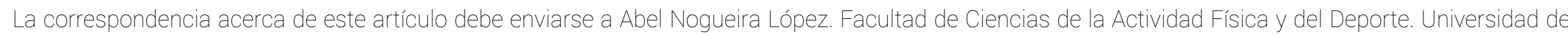
León. Campus Vegazana s/n, 24006 León. E-mail:
} 


\section{Dependência da corrida: uma revisão desde o seu início até a atualidade}

RESUMO: O fenómeno "running" foi e ainda é uma das atividades esportivas que conseguiu mais adeptos nos últimos anos; são muitas as pessoas que saem para correr dia após dia. A corrida converteu-se num comportamento habitual em nossa sociedade ao ser considerada uma das melhores atividades para manter e melhorar a saúde física, mental e psicossocial. O problema surge quando correr converte-senuma necessidade prejudicial, provocando consequências negativas para a saúde e resultando em à Dependência do Exercício - "dependência ou adicçao positiva (APC), quando é uma atividade agradável que gera um prazer extremo", ou "negativa (ANC), quando a vida de uma pessoa é regida por esta atividade". Perante esta situação, o objetivo deste estudo foi determinar o estado de arte da pesquisa sobre a dependência do exercício, com foco na "corrida". A busca foi realizada nas bases de dados Pubmed, Scopus, Web of Science e EBSCOhost usando combinações de palavras-chave: "esporte", "running", "correr" "adicção", "dependência" "maratona". Os principais resultados indicam que a dependência do exercício poderia se incluir na categoria de transtornos do comportamento, porque elacumpre a grande maioria dos critérios de diagnóstico, e nomeadamente pelas graves consequências que pode ocasionar no dia-adia naqueles que a sofrem. Finalmente, propomos algumas recomendações aplicadas que em nossa opinião podem ser consideradas como soluções e alternativas para aumentar o número de pessoas que tiram proveito de todos os benefícios de fazer esporte habitualmente.

PALAVRAS-CHAVE: "Corrida", "adicção", "esporte", "saúde", "revisão".

Artículo recibido: 03/03/2017 | Artículo aceptado: 22/05/2017

Está bien documentado que la realización de actividad física de forma regular, a niveles adecuados, trae consigo indudables beneficios sobre la salud a nivel físico, mental y social. El estudio de dichos beneficios ha centrado la atención de multitud de especialistas por conocer la relación dosis-respuesta, es decir, la frecuencia, la intensidad y la duración de la actividad necesarias para obtener los máximos beneficios posibles.

El término adicción apareció por primera vez asociado al deporte en la literatura científica de la mano de Glasser en 1976, referido a "adicción positiva", como la relación positiva dosis-respuesta entre ejercicio y salud (Berczik et al., 2012; Márquez y de la Vega, 2015). Por otro lado, tres años más tarde, Morgan (1979) acuñó el término de "adicción negativa", para referirse a los efectos tanto a nivel fisiológico (incrementando la posibilidad de sufrir algún tipo de lesión), psicológico (graves alteraciones de la conducta), además de afectar a nivel social, familiar y laboral, en donde las responsabilidades diarias de cada uno de estos ámbitos se podían ver modificadas ante la exposición excesiva que el ejercicio podía llegar a tener (Berczik et al., 2014; Landolfi, 2013). Más recientemente, Antunes, Leite, Lee, Barreto, dos Santos, de Sá Souza y de Mello (2016), han puesto de manifiesto que la práctica regular de ejercicio contribuye tanto a la prevención primaria como a la secundaria de distintas enfermedades crónicas, asociándose a un menor riesgo de muerte prematura. Por otro lado, un gran número de expertos en esta temática (Antunes et al., 2015; De Mello, Aquino, Antunes, Bittencourt, Santos-Silva y Tufik, 2013; Warburton, Nicol y Bredin, 2006); sugieren que el deporte influye de manera muy positiva en el sueño y en el estado de ánimo.

De entre todas las modalidades deportivas estudiadas y analizadas, el "running" destaca respecto a las demás, ya que son muchos los corredores que han experimentado el fenómeno conocido como euforia del corredor o high runner; recompensa neurobiológica, que aparece tanto durante, como después de correr largas distancias y que afecta a nivel central (modulador del estado de ánimo, efecto de bienestar) y a nivel periférico, disminuyendo la sensación de dolor (Dietrich y McDaniel, 2004).

Cuando una actividad inicialmente beneficiosa para la salud, se transforma en adictiva, pueden llegar a producirse incluso síntomas de abstinencia cuando no se puede realizar ejercicio (Hausenblas y Dowms, 2002). Así, en un estudio reciente (Ruiz-Juan y Zarauz, 2016), se han señalado como posibles causas de la conducta adictiva, la combinación de factores socio-culturales como la búsqueda de una imagen corporal ajustada a los cánones de la sociedad; factores fisiológicos, como el papel que juegan las catecolaminas, la activación del sistema opioide endógeno en el cerebro, la regulación de la interlucina-6, y por supuesto factores psicológicos, en donde la mejora del estado de ánimo, la salud, la autoestima, la confianza y las relaciones sociales que se crean con la práctica deportiva regular, hacen que el deporte pueda llegar a convertirse en un comportamiento adictivo (Kerr, Lindner y Blaydon, 2007).

Así, el objetivo general de este artículo es realizar un recorrido en el conocimiento de la adicción al ejercicio y el estado actual, prestando especial atención a la manifestación de este comportamiento en el contexto deportivo del running, para crear un marco actualizado y clarificador de los síntomas y manifestaciones de éste, sumando de esta forma más información para conseguir dar respuesta a uno de los principales retos respecto a este tema, determinar el límite donde la actividad física deja de ser beneficiosa y empieza a deteriorar la salud (Bär y Markser, 2013). 
Según lo citado, en este trabajo, abordaremos el surgimiento del tema tanto a nivel social como científico, su estado actual, características, síntomas más relevantes, el impacto que ha supuesto a distintos niveles en la sociedad, así como, aquellos modelos explicativos que tratan sobre este tema. Para llevar a cabo esta investigación, se han utilizado como fuentes de búsqueda, la información disponible en las siguientes bases de datos, Pubmed, Scopus, Web of Science, EBSCOhost y Google académico, utilizando como palabras clave: "deporte", "running", "correr", "adicción", "dependencia", "marathon", limitando los términos de búsqueda al título, resumen y palabras clave.

\section{Estado en cuestión}

La adicción al ejercicio, ha sido considerada por distintos organismos - American Psychiatric Association en su Manual diagnóstico y estadístico de los trastornos mentales y la World Health Organization (OMS) en la Clasificación Internacional de Enfermedades - como un trastorno de la conducta. Orford (2001), sugiere que la adicción al deporte tiene características similares a las adicciones relacionadas con el alcohol, el juego, la heroína, el tabaco, el comer, el sexo y las más recientes como son internet y las redes sociales (Griffiths, 1995 y 1999). Autores como Weinstein y Weinstein, (2014) señalan como ésta comparte muchos rasgos con los trastornos mentales y conductuales similares, como el predominio de la actividad generadora de la adicción sobre otras actividades, alteraciones emocionales, tolerancia, síntomas de abstinencia y tendencia a una excesiva actividad tras periodos de control.

En 1995, Veale propuso los criterios diagnósticos de este comportamiento (preocupación con el ejercicio estereotipado y rutinario, síntomas significativos de abstinencia en ausencia de ejercicio o alteraciones físicas, sociales, ocupacionales y de otros tipos a consecuencia de la preocupación excesiva por el ejercicio) (Márquez y De la Vega 2015). Por lo tanto, la adicción al ejercicio por sus síntomas, características demográficas, el pronóstico de la enfermedad, la comorbilidad, la respuesta al tratamiento, la prevalencia familiar y su posible etiología, debería ser considerada dentro de las adicciones de tipo conductual (Grant, Potenza, Weinstein y Gorelick, 2010; Griffiths 1996; Hollander y Wong, 1995).

No cabe duda que una de las mayores limitaciones proviene de la confusión conceptual existente ante la gran variedad de términos que se utilizan para referirse y describir las consecuencias de la práctica excesiva de ejercicio. Entre los términos más utilizados encontramos, dependencia del ejercicio (Veale, 1995), ejercicio obligatorio (Pasman y Thomp- son, 1988), ejercicio compulsivo (Yates, 1991) y ejercicio dirigido (Fairburn, 2008), para referirse a las manifestaciones de este tipo de conductas (Johnston, Reilly, y Kremer, 2011).

Respecto a la incidencia de dicha alteración, los estudios se han incrementado considerablemente en los últimos años, así Griffiths, Szabo y Terry (2005), identificaron que el 3,0\% de una muestra de estudiantes británicos de ciencias del deporte y psicología se encontraban en riesgo de adicción al ejercicio, valores que se incrementaban cuando la muestra estudiada incluía atletas de élite o profesionales. Mientras que los resultados obtenidos dos años más tarde por Szabo y Griffiths (2007), utilizando el inventario de adicción al ejercicio (EAI), reflejaron que el 6,9\% de los estudiantes británicos de ciencias del deporte estaban en riesgo de adicción al ejercicio. Valores que, sin embargo, contrastan con el estudio de Allegre, Therme y Griffiths (2007), que únicamente identificaron en una muestra de 95 ultra maratonianos, a tres de estos como en riesgo de adicción al ejercicio (Szabo, Griffiths y Demetrovics, 2016).

Si a estos datos que hace unos años parecían meras hipótesis, le sumamos los valores extraídos de la última encuesta de hábitos deportivos en España (2016), en la que se confirma el aumento de la concienciación que la población española tiene de la importancia de integrar el deporte en su vida - el porcentaje de personas que practicaban deporte en España de manera regular en 2010 , era del 37\%, mientras que, en 2015, este subió hasta el $46.2 \%$-. Estos datos tienen especial relevancia en el colectivo femenino, donde en 2010 sólo el $28.8 \%$ de las mujeres afirmaba realizar deporte semanalmente, frente al $45.4 \%$ de los hombres. Sin embargo, en 2015 estos valores se han igualado hasta el punto que la diferencia entre hombres y mujeres que practican alguna actividad deportiva de forman regular, se ha reducido drásticamente, al aumentar hasta el $42.1 \%$ el porcentaje de mujeres; mientras que los hombres apenas modificaron sus valores, situándose en un $50.4 \%$.

En este cambio de tendencia, el fenómeno del "running" ha sido y continúa siendo uno de los deportes que más adeptos ha conseguido captar, ya que son muchas las personas que de manera regular salen a la calle día tras día a correr. Llegando de esta manera a convertirse en la actividad de ocio más visible desde los años setenta, al tratarse de una actividad de fácil accesibilidad y abierta a todos. Correr se ha convertido en un comportamiento habitual en nuestra sociedad al ser considerado como una de las mejores actividades para promocionar la salud, como señalan Shipway y Holloway (2010). Sin ninguna duda, los corredores son ahora aceptados como un importante grupo de interés dentro de la sociedad. Este auge, está fundamentado principalmente por los numerosos beneficios que correr tiene para la salud, 
ya que permite a sus practicantes disfrutar de la naturaleza y a su vez se convierte en un medio de escape de las tensiones y preocupaciones del día a día. Sin embargo, la paradoja viene motivada por el impacto de los beneficios de la propia actividad tiene en sus participantes, hasta el punto de que, a mayor bienestar, y sensaciones de placer, el riesgo de que aparezcan comportamientos adictivos se multiplica considerablemente.

\section{Aspectos conceptuales}

El concepto de adicción, en sus orígenes y durante años, sólo se utilizó para referirse a el consumo excesivo y descontrolado de sustancias psicotrópicas (Pinna et al., 2015), pero con el paso del tiempo, el abanico de comportamientos incluidos ha ido en aumento, y en la actualidad existe evidencia científica suficiente que lo demuestra, como recoge Griffiths (2005) al señalar como comportamientos adictivos, el juego (Griffiths, 1995), el comer en exceso (Orford, 2001), el sexo (Carnes, 1983), el ejercicio (Terry, Szabo y Griffiths, 2004), los videojuegos (Griffiths y Blackwell, 2002), el uso de Internet (Griffiths, 2000) y el trabajo (Griffiths, 2005).

Marlatt, Baer, Donovan y Kivlahan (1988), definieron el comportamiento adictivo como un patrón de hábitos repetitivos que aumenta el riesgo de enfermedad y/o problemas personales y sociales asociados. Los comportamientos adictivos a menudo se experimentan subjetivamente como "pérdida de control", donde el comportamiento puede ocurrir a pesar de los intentos volitivos de abstenerse o moderar el uso. Estos patrones de hábitos suelen caracterizarse por la gratificación inmediata (recompensa a corto plazo), además de por los efectos deletéreos retrasados (costos a largo plazo) (Griffiths, 2005). Por lo tanto, nos encontramos ante un comportamiento que puede proporcionar placer o alivio del malestar interno (estrés, ansiedad, etc.) y se caracteriza por la incapacidad de controlar el comportamiento (estado de impotencia) y el mantenimiento de éste apesar de las consecuencias negativas (Goodman, 1990).

La manifestación y aparición de este comportamiento no es repentina o de forma abrupta, sino que es más un proceso, caracterizado por la manifestación de seis síntomas que son comunes en todos los adictos y que permiten su identificación (Griffiths, 2005; Modoio, Antunes, Gimenez, Santiago, Tufik y Mello, 2011; Szabo, 2010; Terry et al., 2004):

- Relevancia: Una actividad determinada, en este caso, el ejercicio y más concretamente correr, se convierte en la actividad central del día, en la más importante de todas y domina los pensamientos (preocupaciones), sentimientos y comportamientos, sobre todo evidenciándose en un deterioro o menoscabo de sus relaciones sociales. Correr es lo único que pasa por su cabeza y con lo que asocia todo lo que hace.

- Estado de ánimo: Experiencias subjetivas que la gente tiene como consecuencia de la adicción a una actividad y que interpretan como estrategias de afrontamiento. En el caso de los corredores, la llamada euforia del corredor o Runner's high (Stoll, 1997).

- Tolerancia: Necesidad que provoca incrementar las cantidades de la práctica para lograr los citados efectos placenteros.

- Síntomas de Abstinencia: Sentimientos de malestar y displacer (irritabilidad, estado anímico bajo, etc.) y que pueden provocar efectos de carácter físico cuando no se puede llevar a cabo la actividad o se suceden largos parones injustificados.

- Conflicto: Los problemas que surgen entre la persona adicta y los que conforman su círculo personal/social más cercano (familia, trabajo, vida social, hobbies) por motivo de la actividad a través de la que gira toda su vida.

- Recaída: Repetición de este conjunto de estados o patrones comportamentales de forma casi incontrolable, a pesar de evidenciar algún síntoma de mejoría, sin embargo, son solo avances transitorios.

La adicción al deporte puede llegar a provocar múltiples alteraciones en quienes la sufren, como la generación de niveles elevados de ansiedad, insatisfacción con la imagen corporal, baja autoestima, compulsividad, rigidez, perfeccionismo, extraversión y agradibilidad (Hausenblas y Downs 2002; Hausenblas y Giacobbi 2004; Pugh y Hadjistavropoulos 2011); pérdida de control sobre las actividades de la vida (Griffiths, 1997); pérdida del control sobre el ejercicio (Cockerill y Riddington, 1996); riesgo de auto-lesión (Veale, 1987; Wichmann y Martin, 1992); negación de un problema o auto-justificación (Wichmann y Martin, 1992). Además de una alta necesidad de logro, preocupación por la musculatura, la competencia percibida del ejercicio, autoestima, ejercicio orientado al ego, una pasión obsesiva González-Cutre y Sicilia 2012; Hausenblas y Downs 2002; Paradis, Cooke, Martin y Hall, 2013) y sobre todo, la gran mayoría de los adictos, lo son debido a los motivos de regulación introyectados de cara a la consecución de objetivos (González-Cutre y Sicilia 2012); sentimientos de estrés antes del ejercicio, el ya citado síndrome de abstinencia (a través de la manifestación de la falta de apetito, insomnio, lentitud, fatiga, ansiedad, depresión, culpa, nerviosismo y tensión) después de perder una sesión de entrenamiento (Hausenblas y Downs 2002; Parastatidou, Doganis, Theodorakis y Vlachopoulos, 2014; Zarauz y Ruiz-Juan, 2014)

Es especialmente importante señalar que la adicción al ejercicio, no debe confundirse con el ejercicio que se pro- 
duce a una alta frecuencia, como es el caso de los atletas olímpicos que tienen que dedicarle mucho tiempo a sus entrenamientos y competiciones para poder alcanzar sus objetivos, lo que les conlleva a dedicarle menos tiempo a otras actividades como las relaciones sociales fuera de este ámbito. Pero no por este hecho, estos deportistas se les debe considerar como adictos al deporte (Meyer, Taranis, Goodwin, y Haycraft, 2011).

La adicción a correr se caracteriza especialmente por la aparición de síntomas de abstinencia sufridos durante los períodos de privación (ansiedad, tensión, irritabilidad, espasmos musculares, etc) (Berczik et al., 2014; Egorov y Szabo, 2013; Landolfi, 2013; Márquez y de la Vega., 2015; Parastatidou et al., 2014).

Este fenómeno es considerado parte de un funcionamiento continúo asociado al constructo teórico del compromiso a correr (Carmack y Martens, 1979), y que incluye como variables de estimación el número de días de entrenamiento y el número de kilómetros semanales (Chapman y De Castro, 1990; Ruiz-Juan y Zarauz, 2012; Thornton y Scott, 1995). En el citado estudio Carmack y Martens, desarrollaron y validaron la Escala de Compromiso a Correr (CRS), instrumento para medir los niveles de compromiso de los corredores.

Dos años más tarde, Sachs (1981) analizó el origen y las razones por las que los niveles de compromiso aumentaban o disminuían, llegando a la conclusión de que este comportamiento es "el resultado de la obtención de una mayor calidad de las relaciones sociales, beneficios de salud, estatus social, prestigio y beneficios económicos"; y que se caracteriza por motivos como, correr por la recompensa extrínseca, correr es muy importante, es parte de sus vidas y no pasarlo mal cuando no pueden correr por alguna razón. La importancia de estos motivos es capital ya que, en muchas ocasiones, un elevado compromiso puede generar cierta confusión al tratar de compararlo o igualarlo a la adicción positiva (Glasser, 1976; Morgan 1979). El compromiso, como explica Jaenes (2003), es muy importante cuando se asocia con el deporte, pero especialmente con los corredores de media y larga distancia, porque estos tienen que entrenar cada día volúmenes de entre 80 y $100 \mathrm{kms}$ en los populares y más de $200 \mathrm{kms}$ si hablamos de élite, lo que supone, cuando menos, un gran compromiso.

El Modelo de Compromiso Deportivo de Scanlan, Carpenter, Simons, Schmidt, y Keeler (1993), lo define, "como el constructo psicológico que refleja el deseo y la resolución de persistir en el esfuerzo deportivo durante cierto periodo de tiempo". Además de definir el concepto, en este modelo se proponen los cinco factores que influyen en el deseo de permanecer en una actividad deportiva y que son la diversión (sentimientos generalizados de placer, gusto y diversión ha- cia la actividad deportiva), oportunidades de participación (posibilidades valiosas que se presentan solo a través de una participación continua, por ejemplo, hacer nuevos amigos), alternativas de participación (oportunidades de participar en otras actividades en vez de continuar con el deporte), inversión personal (recursos que se invierten dentro de la actividad) y presión social (expectativas sociales o normas que crean sentimientos de obligación para permanecer en la actividad deportiva).

Otro de los modelos al respecto, es el Modelo de Inversión (Rusbult, Martz y Agner 1998), el cual dirigió todos sus esfuerzos en identificar cuáles son los factores que influyen en el mantenimiento de un comportamiento. Lo que nos viene a decir, es que la satisfacción en una relación o tareas están influenciadas por las recompensas, costos y los resultados esperados por parte de las personas y, en consecuencia, según está valoración el individuo establecerá un nivel u otro de compromiso.

Los maratonianos son los corredores que mayores niveles de compromiso han reflejado, ya que la mayoría de las personas que inician un programa de entrenamiento para correr un maratón, solo un porcentaje muy pequeño abandona, llegando hasta el punto de que el entrenamiento se convierte en parte principal en su día a día, incluso pudiendo afectar a su calidad de vida (Masters, Ogles y Jolton, 1993). Cuando esto sucede, este elevado compromiso se convierte en adicción negativa a correr (NAR), la cual se puede medir a través de La Escala de Adicción a Correr (RAS), instrumento creado por Chapman y De Castro (1990) y validado al castellano por Sancho y Ruiz-Juan (2011). A pesar del componente negativo, correr puede ser una actividad que aumenta la fuerza mental y, si los individuos son privados de ella, sienten algún tipo de dolor, sufrimiento o angustia (física o psicológica), en este caso estaríamos hablando de la conocida como adicción positiva a correr (PAR), definida por Glasser en 1976. Este tipo de adicción positiva, se diferencia de la negativa, por no tratarse de una actividad que domine la vida de la persona, además del sentimiento de placer y agradable asociado a correr.

Por lo tanto, como ya diferenció Pargman (1980), podemos encontrarnos con dos tipos de corredores; los corredores con menor grado de adherencia a la carrera continua, pero con mayor satisfacción al correr (adicción positiva) y, los corredores altamente comprometidos, pero que no suelen disfrutar de correr (adicción negativa). Además del nivel de compromiso que muestren los corredores, se han ido incorporando otras variables que aportan un mayor conocimiento sobre su posible influencia a la hora de considerar la adicción como negativa o positiva. La motivación, la manifestación o no de síntomas de abstinencia, o el número días 
que se sale a correr y la duración de estas sesiones (Antunes et al., 2016; Kanarek, D’anci, Jurdak y Mathes, 2009; Ruíz Juan, Zarauz y Flores-Allende, 2016b; Zarauz y Ruiz-Juan, 2011; Zarauz y Ruiz-Juan, 2012; Zarauz, Ruiz-Juan, Arbinaga, Jaenes y Flores-Allende, 2015).

\section{Modelos Explicativos}

Modelos Fisiológicos. La Hipótesis de la Activación Simpática (Thompson y Blanton, 1987), es un modelo que propone que la adaptación al ejercicio disminuye la actividad simpática del cuerpo, por lo tanto, ante esta menor actividad en reposo, los niveles de activación y excitación consecuentemente también lo serán, no siendo estos suficientes para llevar a cabo muchas de las actividades diarias del día a día. La adaptación al entrenamiento, especialmente aeróbico, genera una disminución de la frecuencia cardíaca y de la actividad simpática que se traduce en un menor nivel de activación. Esta falta de energía, es lo que motiva a las personas a participar en una actividad que aumente su nivel de excitación para lograr un estado óptimo de activación. Por lo tanto, las personas que practican deporte de forma habitual, deberán de aumentar el número de horas e intensidad diaria, para conseguir restablecer su activación (Egorov y Szabo, 2013; Pinna et al., 2015).

El modelo "Interleukin-6" de la Adicción al Ejercicio (Hamer y Karageorghis, 2007) es otra de las hipótesis fisiológicas que gira en torno a la interleucina-6 (IL-6). De acuerdo con esta idea, se considera como un desencadenante no identificado puede causar que los niveles de IL-6 aumenten y generen un comportamiento de enfermedad inducido por citoquinas que está vinculado a un efecto negativo. En individuos afectados por dificultades psicológicas, un nivel elevado de IL-6 podría producir un estado mental aún más negativo. Sin embargo, la hipótesis de la IL-6 puede no explicar la posibilidad de que algunas personas recurran al ejercicio, mientras que otras pueden alcanzar medios químicos de escape. La baja prevalencia de la adicción al ejercicio se atribuye a las posibles adaptaciones al ejercicio, mientras que la falta de ella puede aumentar la vulnerabilidad a la adicción al ejercicio.

Como ya hemos mencionado, una de las hipótesis de más peso, es la encargada de explicar el fenómeno runners' high (Stoll, 1997). La sensación de euforia que sienten muchos corredores tras una carrera intensa y que se ha atribuido a efectos centrales de las endorfinas. El papel que juegan las beta-endorfinas y otros opioides endógenos es el que determina la posibilidad de la aparición o no de los comportamientos dependientes (Adams y Kirkby, 2003). Ha- mer y Karageorghis (2007); Kraemer, et al. (2013), después de estudiar el comportamiento de animales expuestos a diferentes dosis de ejercicio, comprobaron qué ante la exposición continuada a un ejercicio, la sensibilidad a estas hormonas disminuye, por lo tanto, es necesario aumentar la dosis de ejercicio para que sus efectos se mantengan. La intensidad y la duración del ejercicio, son los dos condicionantes que marcan el aumento o reducción de los valores de las endorfinas en el torrente sanguíneo, siendo necesario realizar cualquier actividad deportiva por encima del $60 \%$ del consumo máximo de oxígeno (VO2máx) para que estos valores aumenten (Goldfarb y Jamurtas, 1997). Las encefalinas y dinorfinas (Kraemer et al., 2013), son otras de las hormonas que están implicadas en este proceso y a diferencia de las beta-endorfinas, estas sí que son capaces de atravesar la barrera hematoencefálica. Los estudios realizados con tomografía, han descubierto como la euforia en la que se encuentran los deportistas después de correr, se asocia de manera inversa con diferentes opioides de distintas áreas corticales (Boecker et al., 2008).

El sistema endocannabinoide podría tener también un papel en la adicción al ejercicio, ya que dicho sistema se activa por el ejercicio aeróbico (Dietrich y McDaniel, 2004) y produce efectos de sedación, bienestar y reducción de la ansiedad. Esta hipótesis se demostró después de estudiar el comportamiento en ratones, los cuales redujeron el ejercicio voluntario, después de inhibirles los receptores del cannabinoide tipo-1 (CB1) (Dubreucq, Koehl, Abrous, Marsicano, y Chaouloff, 2010). Según esto parece ser que puede haber una similitud entre el deseo que algunas personas muestran por el ejercicio y el deseo que pueden tener para las administraciones exógenas de cannabinoides. El sistema endocannabinoide también produce sedación, ansiolisis, sensación de bienestar, capacidad atencional reducida, capacidad de memoria de trabajo deteriorada y dificultad en la estimación del tiempo; patrón que encaja casi en su totalidad con el descrito por los corredores de larga distancia, que afirman haber vivenciado en algún momento la euforia del corredor (Dietrich y McDaniel, 2004).

El papel de las catecolaminas, es otra de las hipótesis a tener en cuenta y mantiene que el ejercicio modifica la actividad catecolinérgica cerebral (Cousineau et al., 1977) al formar parte del proceso de regulación emocional y de los sistemas de recompensa. Ambos con una fuerte relación con el inicio y mantenimiento de la práctica deportiva, y consecuentemente se cree que también con la adicción al ejercicio. La mayor limitación de esta teoría, es que para confirmar dichos supuestos se necesita realizar medidas directas en el cerebro. Por el contrario, Lichtenstein, Andries, Hansen, Frystyk y Støving, (2015), resaltaron la existencia de una asociación 
positiva entre la adicción al ejercicio y niveles bajos de leptina en sangre, lo que sugiere una posible implicación de esta molécula en la patogénesis de la adicción al ejercicio, aunque no puede descartarse que sea un resultado del trastorno.

Finalmente, una de las más recientes investigaciones acerca de los marcadores bioquímicos implicados en la adicción al ejercicio ha puesto de manifiesto la existencia de la relación entre ésta y las emociones. Antunes et al. (2016) después de estudiar una muestra de atletas a lo largo de dos semanas sin realizar ejercicio, compararon los efectos de un grupo con síntomas de adicción frente a otro grupo que no mostraba tales síntomas. Para ello utilizaron un acelerómetro actigráfico con el fin de monitorizar el índice de movimiento y midieron la CK y la LDH en muestras de sangre para validar la ausencia de ejercicio, además de una escala para evaluar el estado de ánimo. Los resultados reflejaron como el grupo de adicción al ejercicio mostró un aumento en los niveles de depresión, confusión, ira, fatiga y una disminución generalizada del estado de ánimo, junto con niveles bajos de anandamida y un leve aumento de $\beta$-endorfina post-ejercicio. Dichos resultados, reflejan como el estado de ánimo de los deportistas que muestran comportamientos adictivos, sufre alteraciones significativas.

Modelos Psicológicos. Szabo en 1995 desarrolló la Hipótesis de Evaluación Cognitiva en la que relacionó la variación de los niveles de estrés con el ejercicio. Para este autor, la gran parte de las personas que hacen deporte diariamente, lo realizan con el fin de evadir la tensión, convirtiendo al ejercicio en un elemento vital para poder sobrevivir al estrés de las actividades diarias. El problema surge cuando estas personas no pueden dedicar tiempo al ejercicio, lo que provoca alteraciones a nivel psicológico como los síntomas de abstinencia, perdiendo el control y generando una mayor vulnerabilidad al estrés mediante la aparición de los sentimientos negativos asociados con la falta de ejercicio. Este modelo es criticado al ser capaz únicamente de explicar el mantenimiento de la adicción, pero no su aparición.

Por su parte, Freimuth, Moniz y Kim (2011), propusieron El Modelo de Cuatro Fases para la Adicción al Ejercicio. La primera fase se caracteriza por la actividad placentera mientras que el comportamiento está bajo control. El ejercicio se lleva a cabo desde una perspectiva recreativa buscando la calidad de vida. En la segunda fase, se utiliza el ejercicio como fuente para hacer frente al estrés del día a día, dejando de lado su perspectiva recreativa; mientras que, en la tercera, el deporte se convierte en una actividad diaria y poco a poco va ganando peso y es un problema para incluirlo entre todas las obligaciones diarias, el ejercicio se convierte en la actividad central en torno a la que gira el resto de actividades.
Además, este se pasa a realizar individualmente, en lugar de con amigos, en un equipo, o durante las clases programadas de acondicionamiento físico. La cuarta y última fase es cuando las personas aumentan la frecuencia y la intensidad de la práctica deportiva de forma continua hasta que se convierte en el eje central de su vida y aparecen los síntomas típicos de la adicción plenamente manifestada, incluyendo relevancia, tolerancia, conflicto, necesidad de modificación del estado de ánimo, síntomas de abstinencia y recaída.

Más recientemente McNamara y McCabe (2012) con su modelo "Biopsicosocial", justificaron que la aparición de los comportamientos adictivos al deporte, nace de la interacción de los factores biológicos como, por ejemplo, el índice de masa corporal, los procesos sociales y los psicológicos. Por el contrario, Egorov y Szabo, 2013, presentaron el "Modelo Interaccional", tomando como referencia los factores motivacionales encargados de generar los desencadenantes para el inicio y el mantenimiento de la práctica regular de ejercicio. Según los postulados de este modelo, la adicción al ejercicio, es un síntoma de otra patología psicológica y su manifestación es altamente subjetiva y, por lo tanto, relativamente impredecible.

Otros autores en cambio (Berczik et al., 2014) defienden un modelo interactivo para explicar la adicción al ejercicio muy relacionado con el modelo PACE (Pragmatics, Attraction, Communication, Expectation) elaborado para las adicciones en general (Sussman et al., 2011). Este modelo explica que la motivación para llevar a cabo una actividad física no es más que la interacción de factores personales y situacionales (valores y sociales). Es decir, las personas practican ejercicio por razones de salud o por motivos de rendimiento. Teniendo en cuenta el modelo PACE, ante la posibilidad de la aparición de situaciones estresantes sobre las que se pierde el control, los sujetos intentarán enfrentarse adecuadamente a las mismas (fase pragmática), produciéndose interacciones entre antecedentes, aspectos individuales y factores situacionales (fase de atracción). Llegando incluso a actuar en esta dirección los sujetos orientados al rendimiento, los que se centran más en los aspectos terapéuticos para librarse del estrés (fase de atención) y finalmente, la elección vendrá determinada por la experiencia y creencias de estos, de tal modo que quienes carecen de experiencia con sustancias tóxicas tenderán a enfrentarse al estrés a través del ejercicio (fase de comunicación) (Márquez y de la Vega, 2015; Sussman et al., 2011).

Investigaciones sobre adicción y correr. El número de investigaciones que han estudiado la adicción utilizando una muestra tan específica como la de los corredores populares o amateurs, no es demasiado elevado; por este motivo, se 
han seleccionado aquellas investigaciones más relevantes que utilizan los cuestionarios adaptados al estudio de esta muestra, Running Addiction Scale (Chapman y De Castro, 1990), Commitment to Running Scale (Carmack y Martens, 1979) y Motivations of Marathoners Scale (Masters et al., 1993) todos ellos adaptados al castellano por Zarauz y RuizJuan (2011) para evaluar los niveles de adicción (Escala de Adicción al Corredor, RAS-8), compromiso (Escala del Compromiso del Corredor, CR-11) y motivación (Escala de Motivación de los Maratonianos, MOMS-34) de los corredores.

\section{Running Addiction Scale - Escala de Adicción al Corredor.}

La escala original está compuesta por 11 ítems específicos para medir la adicción al ejercicio en el running y en la que se utiliza una escala de tipo Likert que va desde 1 (no está nada de acuerdo) hasta 5 (está totalmente de acuerdo), para registrar las puntuaciones de preguntas como "Yo no cambiaría actividades con mis amigos para correr" o "Siento que necesito correr al menos una vez al día"; las cuales oscilan entre 11 y 55. La escala tiene una consistencia interna aceptable ( $\alpha=$ 0.82, Chapman y DeCastro, 1990) y la validez ha sido apoyada a través de correlaciones significativas entre las puntuaciones de RAS y la auto-percepción adicción a correr, y entre los valores de RAS y el malestar experimentado cuando no se practica este deporte (Chapman y DeCastro, 1990).

En cuanto a la versión en español, la Escala de Adicción a Correr, fue validada y adaptada por Sancho y Ruiz-Juan (2011), en la cual se redujo el número de ítems a 8 para medir la Adicción Negativa a Correr (ANC) y la puntuación pasó de utilizar una escala tipo Likert de 5 a 7 niveles de respuesta $(1$, no está nada de acuerdo, hasta 7 , está totalmente de acuerdo), de manera que las puntuaciones pueden oscilar entre 8 (mínima ANC) y 56 (máxima ANC). Además, a la hora de realizar los cálculos, los ítems 1, 2, 3 y 4 invierten su puntuación. La consistencia interna de la escala es buena ( $a=$ 0.84 ) y presenta valores correctos que permiten determinar una aceptable bondad de ajuste del modelo original, una vez realizado el Análisis Factorial Confirmatorio $(x 2 / \mathrm{gl}=4.07$; $\mathrm{GFI}=0.88 ; \mathrm{IFI}=0.9 ; \mathrm{CFI}=0.9 ; \mathrm{TLI}=0.88 ; \mathrm{SRMR}=0.05 ; \mathrm{RM}-$ SEA $=0.07$ )

Uno de los primeros estudios llevados a cabo mediante este cuestionario es el de Rudy y Estok (1990), en el que se trata de mostrar el efecto que pueden llegar a tener los comportamientos adictivos sobre las responsabilidades diarias y las relaciones familiares. Para ello se tomó una muestra de 220 corredores de maratón (112 mujeres y 108 hombres) que posteriormente se redujo a 61 mujeres y 55 hombres, al ser los corredores que indicaron en el cuestionario original que estaban casados. La edad de los corredores estaba entre 29 y 71 años $(M=41, D T=9)$, todos ellos habían competi- do como mínimo en una maratón, llevaban entre 4 y 25 años corriendo $(M=8.6, D T=4.2)$ y en donde más del $27,77 \%$ corrían entre 5 y 7 días a la semana.

Para el registro de los datos además de la ya citada RAS, se utilizó The Dyadic Adjustment Scale (DAS) de Spanier (1976), escala compuesta por 32 ítems donde se evalúa la calidad de las relaciones en su día a día, especialmente en las relaciones maritales y que consta de cuatro subescalas (consenso diádico, satisfacción diádica, cohesión diádica y expresión afectiva). De los 32 ítems, 27 de ellos utilizan una escala Likert de 6 niveles de 0 a 5; otros dos ítems una escala de 4 puntos de 0 a 4 ; un ítem con una escala Likert de 0 a 6 y las dos últimas preguntas se puntúan dicotómicamnte (sí = 0 y no = 1). De esta forma las puntuaciones obtenidas pueden oscilar entre 0 y 151, siendo estas últimas las que indican un mayor ajuste. La escala estableció su validez de constructo a través de la comparación con la Marital Adjustmet Scale de Locke-Wallace (1959) en la que para los casados fue de 0.86 y de 0.88 entre los encuestados divorciados $(p<0.001)$. Mientras que la confiabilidad interna es de 0.96 para la escala total y alfas que van desde 0.73 a 0.94 para las subescalas. Los resultados presentados por Rudy y Estok, afirman que correr consume tiempo y energía que generalmente se suele dedicar a la familia, por ello a pesar de que correr es una actividad que mejora la salud, una alta dedicación puede alterar las relaciones familiares.

Años más tarde, en 2003 Aidman y Woollard, examinaron la asociación que los corredores competitivos tienen de su percepción de los posibles niveles de adicción al ejercicio y sus respuestas emocionales y fisiológicas como respuesta a un día de privación de entrenamiento. La muestra estuvo compuesta por 60 corredores, 30 mujeres y 30 hombres con una edad media de 24.2 años, que entrenaban como mínimo cinco veces por semana y que competían en pruebas a nivel regional habitualmente. A estos corredores se les privó de un entrenamiento con una antelación menor de 24 horas sin previo aviso y completaron el Profile of Mood States (POMS) Running Addiction Scale (RAS) y Resting Heart Rate (RHR). En una segunda fase, del total de la muestra fueron seleccionados 15 hombres y 15 mujeres al azar para faltar al siguiente entrenamiento programado, mientras que los 30 corredores restantes continuaron con su entrenamiento. Ambos grupos contestaron el POMS y fue registrado su RHR dentro de las 24 horas posteriores al experimento.

El Profile of Mood States (POMS), (McNair, Lorr, y Droppleman, 1971) se utilizó para registrar las respuestas emocionales derivadas de la privación del ejercicio, ya que esta escala mide diferentes estados de ánimo transitorios como son la tensión, depresión, ira, vigor, fatiga y confusión, junto con la autoevaluación que cada participante tuvo que realizar de su 
frecuencia cardíaca en reposo (RHR), llevando a cabo mediciones diarias, usando un cronómetro en un período de 60 segundos.

Los resultados de este experimento reflejaron como el grupo con privación de ejercicio mostró síntomas significativos de abstinencia a través de las puntuaciones obtenidas en diferentes escalas del POMS, como estado de ánimo deprimido, reducción del vigor y aumento de la tensión, ira, fatiga y confusión, así como un aumento significativo del RHR en las 24 horas posteriores a la sesión de entrenamiento perdida. Aidman y Woollard, constataron como los cambios de humor observados y la respuesta de RHR fueron moderados por la adicción al ejercicio autovalorada y los corredores que tenían puntuaciones inferiores en la RAS, experimentaron significativamente menos cambios de humor y de RHR respecto a los de mayor puntuación en esta escala. Además, las correlaciones entre las puntuaciones de RAS y la magnitud de los aumentos de tensión, ira, confusión, depresión y RHR osciló entre 0.46 y 0.58. En cuanto al grupo control, éste, únicamente mostró alteraciones en el estado de ánimo y RHR. La importancia de estos hallazgos radica especialmente en la percepción que tienen los corredores para auto valorar sus niveles de adicción al ejercicio, moderar sus respuestas emocionales y fisiológicas ante una situación de privación de ejercicio controlado a corto plazo, lo que indica que la magnitud de estas respuestas puede, a su vez, servir como marcadores tempranos de la dependencia del ejercicio.

Otra de las investigaciones que utiliza entre otros instrumentos la escala de adicción a correr, es la llevada a cabo por Smith, Wright y Winrow (2010), en la que analizaron las diferencias en los niveles de dependencia al ejercicio y la ansiedad física social entre corredores competitivos y no competitivos. Para ello dividieron 180 corredores $(M=28.05$, DT = 6.83) en cuatro grupos en función del sexo y si competían o no (masculino competitivo, $n=47$, femenino competitivo, $n=44$, masculino no competitivo, $n=47$, femenino no competitivo, $n=46)$. El hecho de formar parte de una condición competitiva u otra se decidió preguntando en primer lugar a los participantes si se consideraban corredores competitivos, cuando había sido su última carrera, y si se estaban preparando para participar en alguna carrera; siendo los considerados como competitivos todos aquellos que habían participado en alguna prueba durante el último año o estaban planeando competir. Los 180 corredores tuvieron que responder a la Running Addiction Scale (RAS), la Exercise Dependence Scale (EDS-21, Hausenblas y Downs, 2002), medida multidimensional de 21 ítems que registra la dependencia del ejercicio basada en el Manual Diagnóstico y Estadístico de Trastornos Mentales IV (DSM-IV) criterios para la dependencia de sustancias (American Psychiatric Association,
1994). La escala está conformada por siete componentes de la dependencia al ejercicio: tolerancia (como la necesidad de aumentar las cantidades de ejercicio para lograr los resultados deseados o disminuir el efecto de la misma cantidad de ejercicio), síntomas de abstinencia, (la necesidad de falta de más ejercicio), la falta de control sobre el ejercicio, el tiempo (dedicar una gran cantidad de tiempo en las actividades relacionadas con el ejercicio), la reducción de otras actividades, intencionalidad y la continuidad (seguir practicando deporte a pesar de padecer una enfermedad o lesión que nos dificulte su práctica). Para el registro de los datos se eligió una escala Likert de 6 puntos y gracias al cálculo de las puntuaciones de cada componente, se pueden establecer diferencias entre los sujetos en riesgo de dependencia al ejercicio (manifestar tres o más de estos síntomas, lo que es igual a una puntuación 5 o 6 ítem ), los no dependientes-sintomáticos (puntuaciones de 3 a 4) y los dependientes-asintomáticos (puntuación de 1 a 2), además de poder obtener una puntuación global media de los síntomas de la dependencia al ejercicio. Hausenblas y Downs (2002) han demostrado el contenido y la validez concurrente de la escala, a través de una alta confiabilidad interna $(a=0.93)$ y una alta confiabilidad test-retest de 7 días ( $r=$ 0.92). La última de las pruebas que tenían que cumplimentar los participantes fue la Social Physique Anxiety Scale (SPAS), escala unidimensional de 9 ítems que mide la ansiedad relativa a la apariencia física a través de la calificación de cada ítem, "En presencia de otros, me siento aprensivo acerca de mi figura", "Cuando estoy con un traje de baño, a menudo me siento nervioso por la forma de mi cuerpo", en una escala Likert de 5 puntos, pudiendo obtener puntuaciones entre 9 (nada ansioso) a 45 (muy ansioso). La escala ha demostrado la validez del constructo, una fiabilidad aceptable de test-retest $(r=0.82)$ y la consistencia interna ( $a=0.89$; Martin, Rejeski, Leary, McAuley y Bane, 1997).

Los resultados mostraron que los corredores competitivos obtuvieron puntuaciones significativamente más altas en el EDS, reflejando la mayoría de éstos algún tipo de síntomas, al igual que en la RAS; sin embargo, no hubo diferencias significativas respecto a las puntuaciones del SPAS ni a la relación de éste con las otras dos pruebas, lo que supone que los corredores competitivos tienen un mayor riesgo a ser dependientes, pero esto no afecta a sus niveles de ansiedad física social. Además, tampoco encontraron ningún tipo de diferencias entre los hombres y las mujeres.

La última de las investigaciones a destacar en este bloque, es la realizada por Ruiz-Juan y Zarauz en 2012, en la que se partió del total de maratonianos inscritos en la última edición, por aquel momento, de tres maratones muy distantes geográficamente en el territorio nacional (San Sebastián el 30-11-08, Sevilla el 22-02-09 y Barcelona el 01-03-09) y 
con numerosa participación. Mediante un diseño de muestreo estratificado por afijación proporcional, en donde se tuvo en cuenta el sexo (88.94\% hombres; $11.05 \%$ mujeres) y la edad, la muestra aleatoria finalmente estuvo compuesta por 1108 hombres (90.37\%) con rango de edad entre $17 \mathrm{y}$ 71 años $(M=39.92 ; D T=8.91)$ y 118 mujeres $(9.63 \%)$ entre los 21 y 57 años $(M=38.10 ; D T=8.02)$ y entre los que se encontraban desde corredores populares a maratonianos de élite. Ruiz-Juan y Zaraúz dividieron las variables objeto de estudio en dos grupos, por un lado las sociodemográficas (edad, sexo, con quien convive actualmente, número de hijos y/o familiares muy dependientes a su cargo, índice de masa corporal, nivel de estudios y dedicación o actividad laboral y cómo variables de entrenamiento y rendimiento (promedios de kilómetros entrenados a la semana, tiempo por entrenamiento, días entrenados a la semana y compañeros de entrenamiento, porcentaje de tiempo que entrena solo a la semana, años corriendo, tener entrenador, número de maratones terminados, mejor marca en maratón y promedio de marcas en los maratones terminados); mientas que utilizaron el RAS-8, como instrumento para el registro de los datos. Después de los análisis estadísticos, se comprobó como que las diferencias más evidentes entre los hombres que reflejaron puntuaciones más elevadas de ANC (adicción negativa a correr), se encontraron en el mayor número de kilómetros, número de días y horas por entrenamiento a la semana, mayor preocupación por tener un entrenador y conseguir una marca cercana a las 3 horas y media en los 6-7 maratones que ha terminado, frente a una menor preocupación por tener entrenador y ser su primer maratón en los corredores con puntuaciones mínimas en ANC. Mientras que en el caso de las mujeres los resultados obtenidos no fueron los suficiente concluyentes.

Commitment to Running Scale - Escala del Compromiso del Corredor. El compromiso a correr empezó a evaluarse a partir de la elaboración del instrumento de Carmack y Martens (1979), cuestionario compuesto por una escala unidimensional de 12 ítems para medir el grado de compromiso de los maratonianos ante la larga y dura preparación que supone enfrentarse a esta prueba de fondo. El cuestionario constaba de cinco partes - Parte 1 información demográfica, parte 2 cuestiones específicas de la ejecución (la duración y la frecuencia de la carrera, las razones para correr), parte 3 , 22 ítems que describían el estado de ánimo según se iban encontrando (casi nunca, a veces, o casi siempre) en tres partes determinadas de la prueba. En la cuarta parte tenían que responder a los 12 ítems de la Commitment to Running Scale (CR), mediante una escala de 5 puntos, indicando el grado en el que describían sus sentimientos acerca de co- rrer y que en función de su puntuación se les atribuía a los corredores un bajo compromiso (12 puntos) o un alto compromiso (60 puntos) y en la última sección estaban enumerados los 40 beneficios tanto a nivel físico como psicológicos que se le atribuye a correr, y en donde los participantes tenían que señalar con un "sí" o "no", en función de si los habían experimentado o no mientras corrían. El coeficiente de confiabilidad obtenido por Carmack y Martens fue 0.93 y la consistencia interna, medida a través del cálculo de una matriz de correlación entre los 12 ítems de la escala tuvo un coeficiente de correlación intermedia medio de -30.

La adaptación al castellano, igual que en el caso anterior, fue llevada a cabo por Ruiz-Juan y Zarauz (2011) y contiene 11 ítems que miden el compromiso a correr y las costumbres de los corredores con un sistema de respuestas tipo Likert, con puntuaciones que van desde 1 (no está de acuerdo) hasta 5 (está totalmente de acuerdo), lo que permite obtener una puntuación mínima de 11 y una máxima de 55 y en donde los ítems 2, 3, 7, 9 y 10 a la hora de realizar su computación, al igual que sucede en la versión original, es necesario invertir sus respuestas. La consistencia interna de la escala es de $a=0.87$ y presenta valores correctos que permiten determinar una aceptable bondad de ajuste del modelo original.

En cuanto a la literatura más relevante con el uso de este instrumento, es cierto que no existen muchos estudios que lo utilicen, llegando hasta el punto de que en España hasta la adaptación de Ruiz-Juan y Zarauz en 2011, no se había realizado ninguna investigación con estas características, y en la que además de validar la versión al castellano, encontraron que las mujeres puntuaban más alto en el total de la escala (mujeres $M=34.35$; varones $M=33.58$; sobre 55), resultados totalmente en dirección opuesta los del estudio original de Carmack y Martens (1979), donde esta situación se produce a la inversa (hombres $M=48.3$; mujeres $M=45.7$; sobre $60) ;$ además de no encontrar diferencias significativas para la edad. Tal y como se había planteado en las hipótesis, las variables que mejor predicen un mayor compromiso a correr, son el mayor número de kilómetros y el mayor número de días entrenados a la semana.

Con anterioridad a este estudio, en 1992 Acevedo, Dzewaltowski, Gill y Noble, estudiaron las cogniciones y pensamientos de 112 ultra maratonianos que competían en pruebas de 100 millas (aproximadamente 160 kilómetros) utilizando para ello el Sport Orientation Questionnaire, el Trait Sport-Confidence Inventory, la Commitment to Running Scale y un cuestionario diseñado por los investigadores para evaluar las metas, estrategias cognitivas, percepciones de runner's high y los sentimientos derivados de cuando no pueden correr. El Sport Orientation Questionnaire (SOQ; Gill y Deeter, 1988), es 
un cuestionario para evaluar las orientaciones deportivas de logro de los atletas a través de 25 ítems de clasificados en una escala de 5 puntos que van desde muy en desacuerdo hasta muy de acuerdo. La escala evalúa tres constructos: (a) Competitividad, que refleja el deseo de competir y tener éxito en el deporte (13 ítems); (b) Ganar, reflejar el deseo de ganar o evitar la pérdida (6 ítems); y (c) Objetivo, reflejando el deseo de alcanzar estándares de desempeño personal (6 ítems). Gill y Deeter presentaron suficiente evidencia psicométrica para apoyar la fiabilidad (coeficientes alfa de 0.79 a 0.95 ; test-retest de $r=0.73$ a 0.89 ) y la validez de constructo de esta medida. El Trait Sport-Confidence Inventory (TSCI), está compuesto por 13 ítems con una escala de 9 puntos que va desde bajo a alto y con el que se trata de evaluar la creencia o grado de certeza que un individuo posee sobre su habilidad para tener éxito en el deporte (Vealey, 1986). Esta escala presenta una consistencia interna medida por el coeficiente alfa de Cronbach de 0.93 y una fiabilidad test-retest de $r=0.86$ y ha demostrado una validez concurrente y de constructo. Para completar la recogida de datos, Acevedo, Dzewaltowski, Gill y Noble elaboraron un cuestionario en el que se le solicitaba a cada corredor información demográfica, experiencia corriendo, sus objetivos, una meta personal que a mayores tenían que evaluar en función de su importancia y su compromiso, en una escala de 0 (no importante) a 10 (muy importante); junto con las cuestiones relativas al conocimiento de sus cogniciones durante las carreras, en las que se les preguntó a los sujetos si sus pensamientos durante éstas estaban enfocados de manera externa (melodías musicales, problemas en el trabajo, problemas matemáticos, sexo, etc.) o centrados en sensaciones internas (respiración, tensión muscular).

Los resultados de este estudio sugirieron que los ultra maratonianos son un grupo único de atletas, no sólo por el tipo de prueba en la que compiten, sino también por sus orientaciones cognitivas, para las cuales no encontraron diferencias significativas entre los que acaban la prueba y los que no lo consiguen o entre hombres y mujeres. Los pensamientos más recurrentes durante las carreras, estuvieron asociados en su gran mayoría a cogniciones externas, sentimientos de bienestar psicológico y en contadas ocasiones fueron conscientes de experimentar el fenómeno runner's high. Además, la motivación necesaria para competir y completar una carrera de estas características viene dada por la motivación intrínseca, el deseo de alcanzar metas personales y como habían planteado en las hipótesis iniciales, cuando por algún motivo se les privaba de correr, la mayoría mostraba estados psicológicos negativos.

Cinco años más tarde en 1997, Szabo, Frenkl y Caputo se preocuparon por conocer la relación entre adicción, com- promiso a correr y privación de la práctica en una muestra de 100 corredores, los cuales tenían que responder a tres cuestionarios entre los que se encontraban la Commitment to Running Scale, la Obligatory Exercise Questionnaire (Thompson y Pasman, 1991) que mide el grado de adicción al ejercicio, pero a la que le cambiaron la palabra "ejercicio" por "correr", basándose en estudios previos que demostraron que el uso de las palabras "ejercicio", "correr" y "actividad física", de forma indistinta, no afecta a la confiabilidad de la Commit ment to Running Scale. La Obligaroy Exerice Questionnaire, está compuesta por 20 ítems ("Cuando no corro, me siento culpable" o "He tenido sueños relacionados con correr") que se deben puntuar entre 1 (nunca) y 4 (siempre). Mientras que, para evaluar las sensaciones provocadas por la privación de la práctica deportiva, se utilizaron algunos de los ítems (tensión, inquietud, culpabilidad, estrés, irritabilidad, falta de energía, fatiga y peso) de la Running-Deprivation Scale de Robbins y Joseph (1985), como medida para calcular la intensidad de las sensaciones generadas por la falta de ejercicio. Una vez analizadas las puntuaciones de todos los participantes, Szabo, Frenkl y Caputo, llegaron a la conclusión de que la adicción y el compromiso a correr son dos conceptos independientes y la privación de correr, no reflejó ninguna relación con la adicción, pero sí que se mostró como un factor a tener en cuenta con los motivos para seguir practicando este deporte.

\section{Motivations of Marathoners Scale - Escala de Motivación} de los Maratonianos. El instrumento original de Masters et al., (1993), se creó utilizando como base investigaciones previas que se centraron en estudiar los motivos por los que los corredores de larga distancia entrenaban y realizaban esta modalidad deportiva (Carmack y Martens, 1979, Curtis y McTeer, 1981, Masters y Lambert, 1989, Summers, Machin y Sargent, 1983; Summers, Sargent, Levey y Murray, 1982;), a través de los 56 ítems de los que consta este instrumento, los cuales se agrupan en nueve escalas, Orientación a la salud (ítems 8, 14, 17, 26, 37 y 44); Peso (ítems 1, 4, 21, 42); Afiliación (ítems 7, 12, 16, 24, 30, 33); Reconocimiento (ítems 3, 6, 19, 45, 48, 54); Competición (ítems 2, 40, 43, 52); Superación de metas personales (ítems 5, 9, 22, 35, 46, 51); Metas psicológicas (ítems 10, 15, 18, 28, 36, 38, 39, 47, 50); Autoestima (ítems 11, 23, 29, 31, 32, 34, 53, 56) y Significado de la vida (ítems 13, 20, 25, 27, 41, 49, 55). A su vez, estas nueve escalas se distribuyen creando cuatro dimensiones de motivos para correr: Salud física (orientación a la salud y peso), Sociales (Afiliación y reconocimiento), Logro (competición y superación de metas personales) y Psicológicos (metas psicológicas, autoestima y significado de la vida). Cada ítem se valora siguiendo una escala de 1 (no una razón) a 7 (una ra- 
zón importante) en función del grado en el que cada uno de estos representa una razón para entrenar y correr un maratón. La escala cuenta con una consistencia interna adecuada (intervalo de Cronbach alfa de 0.80 a 0.93), una fiabilidad de retest (Rs de intraclase que oscila entre 0.71 y 0.90 ) y una validez factorial de la escala que fue demostrada.

En cuanto a la versión en español, como ya sucedió con los otros dos instrumentos, fue validada y traducida por Zarauz y Ruiz-Juan (2011) y está compuesta únicamente por 34 ítems y 7 escalas: Orientación a la salud (ítems 8, 11, 19, 30); Peso (ítems 1, 4, 15), Superación de metas personales y Compe:ción(ítems 2, 5, 9, 16, 26, 32); Reconocimiento (ítems 3, 6, 13, 31, 33, 34); Afiliación (ítems 7, 10, 12, 17, 21, 24); Meta psicológica (ítems $27,29,28$ ) y Significado de la vida y Autoes:ma (ítems 14, 18, 20, 22, 23, 25). Al igual que en el instrumento original, las respuestas se recogen con una escala de tipo Likert desde 1 (no es una razón para correr) hasta 7 (es una razón muy importante para correr), lo que hace que cada escala presente una puntuación media mínima de 1 (mínima motivación para correr) a 7 (máxima motivación para correr). En cuanto a la consistencia interna de las escalas, esta osciló entre un valor mínimo de a = 0.8 (Orientación a la salud) hasta un máximo de $a=0.9$ (Reconocimiento). Además, el cuestionario presenta valores correctos que permiten determinar una aceptable bondad de ajuste del modelo original.

En cuanto a las investigaciones que utilizaron esta escala como instrumento para la recogida de datos, destaca la realizada por Doppelmayr y Molkenthin (2004), quienes se interesaron por estudiar y comparar las fuentes de motivación de los corredores de maratón y de ultra maratón, concretamente los que habían participado en el Maratón de los Sables (MdS), una maratón por el desierto que se desarrolla durante 6 días-etapas y con una longitud total de $230 \mathrm{~km}$. Debido a la escasa participación femenina, los datos utilizados para elaborar el estudio se centraron exclusivamente en los sujetos masculinos (35 corredores de maratón, 60 corredores ultra y 54 participantes MdS). Los resultados demostraron que los motivos por los que las personas que participan en carreras de mayor distancia que una maratón, son distintos a los de los maratonianos, para los cuales, la naturaleza y el significado de vida son los motivos de mayor importancia y la Competición el motivo menos valorado al contrario que los maratonianos.

Desde 2011, el volumen de investigaciones que utiliza esta escala ha crecido considerablemente, por ejemplo, Krouse, Ransdell, Lucas y Pritchard estudiaron diferentes aspectos de las 344 mujeres que participan en carreras de ultra maratón, entre los que se encuentran sus motivaciones, objetivos, hábitos de entrenamiento o tener entrenador, variables ya utilizadas en otras investigaciones del estilo de esta y sobre todo, variables que influyen directamente en la consecución de unos u otros resultados. Además del MOMS, también se utilizó para medir los objetivos de estas ultra maratonianas, el Perception of Success Questionnaire (PSQ) cuestionario con una validez de contenido establecida a través de la evaluación repetida por un panel de expertos compuesto por investigadores de la motivación y psicólogos del deporte y en la que los alfas de Cronbach para las escalas tarea y ego fueron 0.89 y 0.90 , y la fiabilidad test-retest fue 0.97 y 0.98 respectivamente. Los hallazgos más importantes de este estudio fueron que muchas de estas mujeres entrenaban más de 12 horas semanales, a pesar de tener un trabajo a jornada completa y tener a su cargo el cuidado de niños. Éstas, son mujeres que tienen como principales fuentes de motivación la salud y el logro y están más orientados a la tarea que al ego, y que por norma general no suelen contar con los servicios de un entrenador, debido al coste o que simplemente consideraban que no lo necesitaban. Un año más tarde, fueron Heazlewood et al. (2012), estudiaron la eficacia de esta escala (MOMS) a la hora de evaluar los factores motivacionales en diferentes deportes, siguiendo los estándares éticos de la Declaración de Helsinki. Los resultados obtenidos después de la cubrir el cuestionario MOMS en línea, por parte de 1590 atletas (739 varones, 851 mujeres) de 25 a 83 años $(M=49.1$ años, $D T \pm 9.0)$ que participaron en los Juegos Panamericanos de 2010, demostraron que las pruebas de Cronbach respecto a las 9 escalas del instrumento de afrontamiento, autoestima, sentido de vida, salud, preocupación por el peso, afiliación, reconocimiento y logro de metas personales tenían valores superiores a 0.8 mientras que la escala de rendimiento tenía un valor mayor que 0.7. El análisis factorial confirmatorio utilizando la extracción de máxima verosimilitud y una serie de rotaciones reveló que la estructura del factor MOMS fue parcialmente apoyada para esta diversa población de atletas de ambos sexos. Sin embargo, hubo algunas discrepancias en cuanto a la complejidad de factores en diez ítems y algunas cargas de factores inversos para los constructos de afrontamiento psicológico, autoestima y significado de vida.

Como ya sucedió con los otros instrumentos, Ruiz-Juan y Zarauz (2012), también se han interesado por el estudio de las motivaciones de los corredores de maratón españoles. Para ello administraron la Motivations of Marathoners Scale-34 a una muestra de 1226 corredores de 3 maratones, 1108 hombres con rango de edad de 18 a 71 años $(M=39.92$; $D T=8.91)$ y 118 mujeres con rango de edad de 21 a 57 años $(M=38.10 ; D T=8.02)$ y donde obtuvieron que las motivaciones más altas fueron intrínsecas o relacionadas con la tarea (significado de la vida-autoestima y orientación a la sa- 
lud) y las más bajas extrínsecas o relacionadas con el ego (reconocimiento) lo que según estos autores puede explicar en gran parte que los maratonianos mantengan su práctica deportiva durante gran parte de su vida (Masters et al., 1993), motivos los cuales están en un continuo proceso de evolución de la mano de la edad y el número de maratones terminados. Además de obtener otros resultados menos esperados como que las mujeres puntuaron significativamente más alto que los hombres en 4 de las 7 escalas, tendencia que sigue aumentando en la actualidad.

En 2015, son varias las investigaciones que tomaron esta escala (MOMS), como base para la recogida de datos y como ya ha ocurrido con otros estudios anteriormente citados, el papel de la mujer en este tipo de investigaciones cobra especial relevancia, así Lendrum (2015), se decidió por explorar las experiencias por las que pasan las mujeres maratonianas. Para ello utilizó una entrevista y la ya citada escala de motivación para los maratonianos (MOMS) que completaron 20 corredoras de maratón estadounidenses principalmente de la región del Medio Oeste de los Estados Unidos, 10 mujeres de raza blanca y 10 de raza negra entre 30 y 70 años, grupo de edad promedio de las mujeres que corren maratón en los Estados Unidos. Las conclusiones obtenidas muestran como este grupo de mujeres basan su participación en estas pruebas de forma recreativa y no competitiva, corren por motivaciones diversas, como son la salud y el estado físico, la salud mental, la resolución de problemas, el componente social de la carrera, el logro de objetivos, la libertad y la autoconfianza. Lendrum, pudo constatar también que las mujeres que forman parte en estos eventos experimentan restricciones de género y sentimientos de culpa asociados con su participación en el deporte. Por su parte, Zach, Xia, Zeev, Arnon, Choresh y Tenenbaum (2015), probaron y ampliaron el modelo Motivation of Marathoners Scale (MOMS) con el fin de examinar la evidencia para lograr la mejor solución respecto a la validación transcultural del modelo, distribuyéndolo a 306 corredores de maratón masculinos y femeninos con una edad entre 20-77 años y que hubiesen corrido ya algún maratón. Después de realizar los pertinentes análisis factoriales exploratorias y confirmatorias, se demostró que la solidez psicométrica del nuevo modelo MOMS no sólo supera al modelo anterior, sino que también demuestra esencialmente lo que el primero no pudo hacer. El pobre ajuste del modelo original confirmó que la naturaleza de los motivos para correr un maratón no está orientada jerárquicamente con factores de primer orden y de segundo orden; mostrando que, todos los factores podrían considerarse mejor como factores independientes. Además, este nuevo modelo distinguió dos factores adicionales respecto a las 9 escalas del instrumento. Para el Afrontamiento psicológico (afrontamiento emocional y el manejo de la vida cotidiana) uno solo en la versión original, Orientación general de la salud (reducción de la prevalencia de la enfermedad y la longevidad y mantenerse en forma) sólo un factor en el modelo original. Zach y colaboradores argumentan a favor de este nuevo modelo qué si bien el primero no se basa en ningún marco teórico, éste lo hace tomando como referencia la Self-Determination Theory (SDT; Deci y Ryan, 1985). No obstante, y a pesar de todas las evidencias a favor del modelo actual, recomiendan comprobar y verificar las propiedades psicométricas del modelo en otras culturas y utilizar otras variables relativas a la muestra como el nivel de competencia, género, número de años corriendo, etc. Una investigación más llevada a cabo en este año, fue la de Larumbe, Pérez-Llantada, López de la Llave y Buceta (2015), en la que desarrollaron un instrumento en español con el fin de medir y conocer el estado psicológico de los corredores de maratón durante los días y horas previos a la carrera. Para ello diseñaron una prueba compuesta por 3 estudios; en el primero participaron 1060 corredores aficionados de entre 18 y 57 años y el análisis factorial exploratorio mostró cinco dimensiones (motivación, autoconfianza, ansiedad, percepción del estado físico y apoyo social percibido). Los otros dos estudios posteriores se encargaron de examinar las propiedades psicométricas de una versión depurada de esta medida. Además, en el segundo estudio, 801 corredores aficionados (17-63 años) de una muestra independiente completaron el cuestionario y el análisis factorial confirmatorio apoyó un modelo de seis factores. La consistencia interna fue de 0.72 a 0.90 . Finalmente, en el estudio 3 , una muestra independiente de 22 corredores aficionados de entre 28 y 47 años cumplimentaron la versión final de PODIUM de 20 ítems y el MOMS y otra muestra compuesta por $36 \mathrm{co}-$ rredores también aficionados (23-57 años) cubrieron el PODIUM y la versión más reciente de la adaptación al español del CSAI-2 (Andrade et al., 2007), para medir la ansiedad y auto-confianza. En apoyo de la validez concurrente del PODIUM, la escala de motivación correlacionaba con el MOMS, y las escalas de ansiedad y auto-confianza correlacionaban con el CSAI-2.

El último estudio al que hacemos referencia en este apartado es el llevado a cabo por Zarauz, Ruiz-Juan y Flóres-Allende (2016), los cuales se preocuparon por estudiar las motivaciones que hacen que los sujetos estén motivados para realizar ejercicio físico, concretamente estudiaron cuáles son las relaciones predictivas entre los motivos a correr y varios hábitos de entrenamiento de los maratonianos. Para ello seleccionaron una muestra de 1795 corredores de fondo en ruta, de los cuales 1105 eran españoles y 690 mexicanos, con un total de 1541 hombres con un rango de edad 
de 18 a 76 años $(M=38.98 ; D T=10.45)$ y 254 mujeres entre 18 y 69 años $(M=37.88 ; D T=9.80)$, de entre todos los corredores inscritos en las ediciones de 2011 de la media maratón de Almería y Elche (España) y en la edición de 2011 y 2012 de la media maratón de Guadalajara (México), junto con las respuestas recibidas a través de una página web habilitada con tal fın, a través de la que se obtuvieron 741 cuestionarios de corredores de maratón y medio maratón desde 01/03/11 a 20/03/13.

Las conclusiones a las que llegaron fueron que el número de corredores que participan en este tipo de pruebas cada vez es mayor, y la participación les ayuda a mejorar su autoestima, su salud y darle un sentido a su vida, sobre todo en el caso de las mujeres, las cuales afrontan este reto con un mayor compromiso y seriedad que los hombres. Además, se obtuvieron siete sólidos modelos predictivos de las motiva- ciones de los corredores (Orientación a la salud, Peso, Metas personales-Competición, Reconocimiento, Afiliación, Metas psicológicas, Significado de la vida-Autoestima), que se pueden predecir mediante variables muy diferentes, de ahí la importancia que tiene el realizar en los análisis pruebas en las que se haga una diferenciación por sexos.

Para finalizar con este apartado se presentan dos tablas resumen. En la Tabla 1, se han recogido las investigaciones realizadas con las versiones adaptadas al español por RuizJuan, Zarauz y colaboradores, en las que han utilizado más de uno de los instrumentos explicados anteriormente; mientras que en la Tabla 2 se pueden observar de manera esquemática las características fundamentales de otras investigaciones no citadas hasta el momento, pero no por ello menos importantes, las cuales utilizan una muestra de corredores o la adicción como objeto de estudio.

Tabla 1. Investigaciones sobre adicción en castellano (CR-11, RAS-8 y MOMS-34)

\begin{tabular}{|c|c|c|}
\hline Autores & Muestra & Contenido \\
\hline Zarauz y Ruíz-Juan (2011) & 1226 Maratonianos & $\begin{array}{l}\text { Aclarar por qué se pasa de ser un } \\
\text { corredor comprometido a ser un } \\
\text { corredor adicto negativamente a } \\
\text { correr y qué variables específicas los } \\
\text { predicen }\end{array}$ \\
\hline Zarauz y Ruíz-Juan (2012) & 1226 Maratonianos & $\begin{array}{l}\text { Analizar qué variables motivacio- } \\
\text { nales y de entrenamiento predicen } \\
\text { el elevado compromiso y adicción } \\
\text { negativa de los maratonianos para } \\
\text { tratar de obtener unas conclusiones } \\
\text { útiles para aumentar la adherencia a } \\
\text { programas de actividad físico-depor- } \\
\text { tiva en la población general.x }\end{array}$ \\
\hline
\end{tabular}

Zarauz, Ruíz-Juan, Arbinaga, Jaenes y Flores-Allende (2015)
1795 Corredores de Fondo
Conocer qué relaciones predictivas presentaban el compromiso a correr, la ansiedad competitiva y la percepción de éxito en las carreras en ruta sobre los motivos para realizarlas. Además de buscar la posible existencia de influencias culturales en la contextualización de estos modelos entre corredores españoles y mexicanos.
Variables

Sociodemográficas (edad, género, $n^{0}$ hijos, pesos, estudios, situación laboral, etc.) y de Rendimiento (kms por semana, horas de entrenamiento, años corriendo, etc.)

Sociodemográficas (edad, género raza, con quien convive, número de hijos y/o personas muy dependientes a su cargo, índice de masa corporal, nivel de estudios y dedicación o actividad laboral) y de Rendimiento (promedios de kilómetros entrenados por semana, horas por entrenamiento, días de entrenamiento por semana y años corriendo)
Instrumentos de medida

CR y RAS

CR, RAS y MOMS

CR, MOMS, CSAI-2R y POSQ
Hábitos de entrenamiento ( $k m s$ por semana, tiempo por entrenamiento, años corriendo, índice de masa corporal, etc.)

\section{CR y RAS}

to, el grado de compromiso y el de adicción negativa.
Ruíz-Juan, Zarauz y Flo- 1795 Corredores res-Allende (2016b)
Medio Maratón 
Tabla 2. Algunas investigaciones relevantes sobre la adicción al deporte

\begin{tabular}{|c|c|c|c|}
\hline Autores & Muestra & Contenido & Instrumentos de medida \\
\hline Sanz, Blasco y Cruz (1992) & - & $\begin{array}{l}\text { Descripción y análisis del concepto de acti- } \\
\text { vidad física de fondo (euforia del corredor, } \\
\text { tolerancia, síndrome de abstinencia...) }\end{array}$ & - \\
\hline Furst y Germone (1993) & 188 Popular & $\begin{array}{l}\text { Relación adicción negativa con variables } \\
\text { como sexo, edad, años practicando depor- } \\
\text { te, etc. }\end{array}$ & Demographic Scale,NAS \\
\hline $\begin{array}{l}\text { Slay, Hayaki, Napolitano y } \\
\text { Brownell (1998) }\end{array}$ & 323 Corredores & $\begin{array}{l}\text { Estudiar la asociación entre los motivos } \\
\text { para correr y los hábitos de alimentación }\end{array}$ & $\begin{array}{l}\text { ORQ, The Aberdeen Marathon Follow-Up } \\
\text { Questionnaire, EAT }\end{array}$ \\
\hline Hulley y Hill (2001) & 226 Corredoras Élite & $\begin{array}{l}\text { Examinar la presencia de trastornos de } \\
\text { alimentación, asociado al tipo de entrena- } \\
\text { miento, dieta, salud, etc. }\end{array}$ & $\begin{array}{l}\text { EDE-Q, Mental Health Inventory, Rosen- } \\
\text { berg's Self-Esteem Questionnaire y Body } \\
\text { Cathexis Scale }\end{array}$ \\
\hline $\begin{array}{l}\text { Bueno, Capdevila y Fernán- } \\
\text { dez-Castro (2002) }\end{array}$ & 135 Atletas & $\begin{array}{l}\text { Estudia la relación de aspectos precom- } \\
\text { petitivos, como la ansiedad, el estado de } \\
\text { ánimo y la autoeficacia, y aspectos acae- } \\
\text { cidos durante la competición, como las } \\
\text { percepciones de amenaza y los recursos } \\
\text { de afrontamiento, con el rendimiento }\end{array}$ & $\begin{array}{l}\text { Cuestionario Precompetitivo de Datos } \\
\text { Censales, Deportivos y Demográficos; } \\
\text { CSAI-2; POMS; CAMDER; CADER; }\end{array}$ \\
\hline
\end{tabular}

$\begin{array}{ll}\text { Buceta, López de la Llave, } & 1220 \text { Corredores Popu- } \\ \text { Pérez-Llantada, Vallejo y } & \text { lares } \\ \text { Del Pino (2003) }\end{array}$

Del Pino (2003)
Análisis del estado psicológico de corredores populares de maratón 65-12 horas antes de la prueba, en tres variables relevantes: ansiedad-estado somática, ansiedad-estado cognitiva y autoconfianza-estado.

\section{CSAI-2}

$\begin{array}{lll}\text { Szabo y Griffiths (2006) } & \begin{array}{l}445 \text { Estudiantes Ciencias } \\ \text { del Deporte y Población } \\ \text { Deportista General }\end{array} & \begin{array}{l}\text { Comparar la prevalencia de síntoma de } \\ \text { adicción al ejercicio entre estudiantes de } \\ \text { ciencias del deporte y población general } \\ \text { activa. }\end{array}\end{array}$

Arbinaga y Caracuel (2007) 116 Fisioculturistas

Conocer la existencia o no de comportamientos adictivos en una muestra hasta el momento sin estudiar

Escala de Adicción General Ramón y Cajal

PVS

Obtener un instrumento apropiado para la evaluación de la Personalidad Resistente

\section{en maratonianos \\ Jaenes, Godoy y Román 189 Maratonianos (2008)}

Bueno, Weinberg, Fernandez-Castro y Capdevila (2008)

35 Atletas de resistencia Larumbe, Pérez-Llantada y 8 Corredores Populares López de la Llave (2009)
De la Vega, Rivera y Ruiz
130 Corredores
corredores (2011)
Estudiar los mecanismos psicológicos que están implicados a la hora de marcar objetivos en los deportistas de resistencia
AITES, AICRES, Self-Efficacy Scale

Entrevista Semiestrucutrada

Identificar las variables psicológicas más representatvias de los maratonianos populares, horas antes de la carrera
Comparar los niveles de resiliencia entre dos grupos de corredores de resistencia y determinar si existe correlación con el rendimiento en carrera. 
Tabla 2. (Continuación)

\begin{tabular}{|c|c|c|c|}
\hline Autores & Muestra & Contenido & Instrumentos de medida \\
\hline Lane y Wilson (2011) & 34 Corredores & $\begin{array}{l}\text { Investigar las relaciones entre la inteli- } \\
\text { gencia emocional rasgo y los cambios del } \\
\text { estado emocional en el transcurso de una } \\
\text { carrera ultra-resistencia }\end{array}$ & BRUMTS, UWIST, EIS \\
\hline $\begin{array}{l}\text { Burtscher, Furtner, Sachse y } \\
\text { Burtscher (2011) }\end{array}$ & $\begin{array}{l}127 \text { Corredores de } \\
\text { montaña }\end{array}$ & $\begin{array}{l}\text { Validar al alemán la Escala de Motivación } \\
\text { del Deporte (SMS28) e investigar las } \\
\text { diferencias de sexo, edad respecto a la } \\
\text { motivación de corredores de montaña. }\end{array}$ & SMS-28 \\
\hline $\begin{array}{l}\text { Salazar, Castro, Remicio, } \\
\text { Zambrano y Colombia } \\
\text { (2012) }\end{array}$ & 30 Fisioculturistas & $\begin{array}{l}\text { Determinar el nivel de adicción en relación } \\
\text { al ejercicio corporal }\end{array}$ & $\begin{array}{l}\text { Escala de Adicción General Ramón y } \\
\text { Cajal }\end{array}$ \\
\hline $\begin{array}{l}\text { Szabo, De la Vega, Ruiz-Bar- } \\
\text { quín y Rivera (2013) }\end{array}$ & $\begin{array}{l}242 \text { Estudiantes Univer- } \\
\text { sitarios }\end{array}$ & $\begin{array}{l}\text { Influencia del sexo, contexto social y nivel } \\
\text { deportivo en la aparición de la adicción al } \\
\text { ejercicio }\end{array}$ & EAl \\
\hline Cook et al. (2013) & 2660 Corredores & $\begin{array}{l}\text { Examinar los niveles de dependencia al } \\
\text { ejercicio y las diferencias entre los sínto- } \\
\text { mas de la dependencia primaria y secun- } \\
\text { daria. }\end{array}$ & EXD \\
\hline $\begin{array}{l}\text { Zingg, Knechtle, Rüst, } \\
\text { Rosemann, Lepers (2013) }\end{array}$ & 64100 Maratonianos & $\begin{array}{l}\text { Estudiar los cambios en el rendimiento } \\
\text { en función del género de corredores que } \\
\text { compiten en una maratón de montaña } \\
\text { frente a los que participan en una maratón } \\
\text { en la ciudad. }\end{array}$ & $\begin{array}{l}\text { Ecuaciones de Cálculo del Ratio de Rendi- } \\
\text { miento }\end{array}$ \\
\hline $\begin{array}{l}\text { Ruíz-Juan, Zarauz y Arbina- } \\
\text { ga (2013) }\end{array}$ & 401 Corredores & $\begin{array}{l}\text { Validación de la Escala de Adicción al } \\
\text { Entrenamiento }\end{array}$ & EAE \\
\hline Zarauz y Ruíz-Juan (2014) & 401 Corredores & $\begin{array}{l}\text { Estudiar la relación entre la ansiedad } \\
\text { precompetitiva y los niveles de motivación, } \\
\text { adicción, satisfacción intrínseca, percep- } \\
\text { ción y creencias sobre las causas del éxito } \\
\text { en el deporte. }\end{array}$ & EAE, SMS, CSAI-2R, POSQ, SSI, BACSSQ \\
\hline $\begin{array}{l}\text { Youngman y Simpson } \\
\text { (2014) }\end{array}$ & 1285 Triatletas & Riesgo de sufrir adicción al ejercicio & EAI \\
\hline $\begin{array}{l}\text { Lichtenstein, Larsen, Chris- } \\
\text { tiansen, Stoving y Bredahl } \\
\text { (2014) }\end{array}$ & 274 Deportistas & $\begin{array}{l}\text { Comparar la prevalencia de adicción al } \\
\text { ejercicio entre deportes individuales y } \\
\text { colectivos }\end{array}$ & EAl \\
\hline Summers (2015) & - & $\begin{array}{l}\text { Diferencias entre adicción, pasión y deseo/ } \\
\text { apetitos }\end{array}$ & - \\
\hline $\begin{array}{l}\text { Konkolÿ, Woodin, Hodgins y } \\
\text { Williams (2015) }\end{array}$ & - & $\begin{array}{l}\text { Prevalencia del consumo de sustancias, } \\
\text { comorbilidad y efecto de estas a lo largo } \\
\text { de } 5 \text { años en } 6 \text { comportamientos exce- } \\
\text { sivos (ejercicio, sexual, compras, video } \\
\text { juegos, comer y chat online) }\end{array}$ & $\begin{array}{l}\text { Quinte Longitudinal Study, Composite } \\
\text { International Diagnostic Interview, Alco- } \\
\text { hol, Smoking and Substance Involvement } \\
\text { Screening Test y Problem and Pathologi- } \\
\text { cal Gambling Measure }\end{array}$ \\
\hline
\end{tabular}


Tabla 2. (Continuación)

\section{Autores}

Griffiths, Urbán, Demetrovics, Lichtenstein, De la Vega, Kun, Ruiz-Barquín, Youngman y Szabo (2015)
Muestra

6031

Cernuda, De la Gándara y $\quad 408$ Alonso (2015)

Fernández Macías, Godoy-Izquierdo, Jaenes, Bohórquez y Vélez (2015)
Contenido

Análisis de las características psicométricas de la Escala de Adicción al Ejercicio con una muestra de 5 países diferentes
Instrumentos de medida

EAI

SAS-40

Elaboración de la Escala SAS-15 (Sport Addiction Scale-15) y la obtención de sus propiedades psicométricas.
170 Maratonianos

Explorar si corredores de maratón con diferentes niveles de experiencia experimentaron estados de "flow" y las características de este fenómeno en esta población atlética. Además de analizar la relación con este concepto flujo los tiempos esperados y logrados en las últimas carreras

\section{EBF, FSS-2, DFS-2}

Importancia de las diferencias de sexo en el estudio de la adicción

Becker, McClellan y Glover Reed (2016)

De la Vega, Parastatidou, 313 Deportistas Ruíz-Barquín y Szabo (2016)

Macfarlane, Owens y Del Pozo (2016)
10 Expertos de diferentes ámbitos del deporte
Relación entre adicción al ejercicio y la pasión (harmoniosa y obsesiva)

\section{EAI, SPS}

Establecer un consenso en las herramientas de adicción al ejercicio e identificar como estas se ajustan al model adaptado de adicción al ejercicio "Work Craving Model"

\section{Metodología Delphi}

Estudiar los síntomas de retirada y abstinencia

Entrevista Semiestrucutrada

Heirene, Shearer, Rode- 4 Escaladores rique-Davies y Mellalieu (2016)
Ruíz-Juan y Zarauz (2016) 401 Corredores
Conocer los niveles de adicción al entrenamiento, motivación, ansiedad, satisfacción intrínseca, percepción y creencias sobre las causas del éxito en el deporte

\begin{tabular}{ll}
\hline $\begin{array}{l}\text { Ruíz-Juan, Zarauz y Flo- } \\
\text { res-Allende (2016a) }\end{array}$ & Estudiar los efectos predictivos de la \\
& ansiedad precompetitiva en relación con \\
& otras variables que entran en juego en las \\
& carreras de larga distancia
\end{tabular}

EAE, SMS, CSAI-2R, POSQ, SSI, BACSSQ

MacNicol (2017)

Revisión sobre los mecanismos neurobiológicos que están implicados en el abuso de sustancias y la adicción, prestando especial atención a los mecanismos que favorecen su aparición y la recaída

Johnson y Lovinger (2016)

Revisión sobre tres de los neurotransmisores Dopamina (DA; D1-D2), Endocanabinoides (eCBs; CB1 receptores) y Glutamato [grupo II receptores del glutamato metabotrópico (mGlu)] 
Tabla 2. (Continuación)

\begin{tabular}{|c|c|c|c|}
\hline Autores & Muestra & Contenido & Instrumentos de medida \\
\hline Suckling y Nestor (2017) & & $\begin{array}{l}\text { Revisión sobre el papel de la MRI (Imagen } \\
\text { por Resonancia Magnética) y la importan- } \\
\text { cia futura de la utilización de las mejoras } \\
\text { tecnológicas para avanzar en los estudios } \\
\text { de adicción }\end{array}$ & \\
\hline
\end{tabular}

\section{Discusión}

El ejercicio regular es una práctica adecuada para mantener y mejorar la salud física y mental, sin embargo, realizar ejercicio de forma excesiva puede llegar a generar graves problemas de salud y dificultades para lograr los ansiados beneficios positivos del deporte. Las investigaciones al respecto, cifran en un 3\%, el porcentaje de personas que están en riesgo de ser adictos (Márquez y de la Vega, 2015). No obstante, debido a la falta de investigaciones que relacionen la adicción y el running; se antoja necesaria la validación de instrumentos, la confirmación de unos criterios diagnósticos y el estudio de la comorbilidad de este patrón conductual con otros comportamientos de riesgo como los hábitos de alimentación o determinados rasgos de personalidad, lo que podría suponer un aumento en el número de personas adictas.

Es un hecho qué a la luz de los resultados de las investigaciones más recientes, se muestra que estamos ante una nueva realidad, que puede llegar a convertirse en un problema preocupante en la sociedad actual. Por lo tanto, los esfuerzos de las investigaciones futuras en este campo, deben tener como primer objetivo, describir, delimitar y conceptualizar este emergente "trastorno de la conducta" y tratar de encontrar y dirigir la práctica deportiva hacia la salud. Generar hábitos orientados a la creación de una motivación, compromiso y adicción positiva, que permita la realización de una práctica deportiva controlada y orientada al placer y al disfrute sin que este se convierta en un obstáculo para la consecución de nuestros objetivos. Donde la posibilidad de disponer de un entrenador y/o pertenecer a un grupo, club o escuela de corredores populares puede ser uno de los primeros pasos para fomentar la práctica saludable.

\section{Aplicaciones Prácticas}

Al tratarse de un tema tan específico y en el que muchos de los conceptos analizados no están todo lo claros posibles, no es fácil determinar, más allá de las estrategias ya conocidas para otros trastornos adictivos (Stoll, 1997), las pautas más adecuadas para poder orientar a los deportistas hacia una práctica saludable. Además, al tratarse de una realidad relativamente nueva, son necesarios muchos más estudios que ayuden a clarificar y sobre todo que aporten datos e información más concisa sobre la manifestación de este comportamiento para poder actuar y poner en marcha las estrategias y herramientas más propicias para alcanzar los objetivos deseados.

No obstante, al encontrarnos delante de una situación que afecta no solo al propio sujeto que la padece, sino a todos los que lo rodean, el papel que tiene el círculo más cercano a este tiene una importancia vital a la hora de reducir este tipo de comportamientos, junto con la necesidad de buscar asesoramiento de todos los especialistas del running, entrenadores, fisioterapuetas, médicos, nutricionistas, psicólogos del deporte, etc., con el fin de tener la mayor información posible y de esta forma reducir las malas prácticas.

En concreto, desde una perspectiva profesional especializada, el psicólogo del deporte (Cantón, 2016) puede contribuir a la intervención en el ámbito de la adicción a correr desde una perspectiva interdisciplinar. Por último, es fundamental que esta afición no se convierta en el único sustento a nivel motivacional y emocional en torno al que gira la vida de las personas que la practican, porque en ese caso, los éxitos y los fracasos derivados de la carrera, serán los que determinen el estado de ánimo y los comportamientos de estos respecto al resto de ámbitos de su vida. El deporte y concretamente correr, debe de ser uno de los pilares, pero bajo ningún concepto el único.

\section{Referencias}

Acevedo, E., Dzewaltowski, D., Gill, D. y Noble, J. (1992). Cognitive orientations of ultramarathoners. The Sport Psychologist, 6, 242-252.

Adams, J. y Kirkby, R. (2003). El exceso de ejercicio como adicciónn: una revisión. Revista de toxicomanías, 34, 10-22.

Aidman, E. y Woollard, S. (2003). The influence of self-reported exercise addiction on acute emotional and physiological res- 
ponses to brief exercise deprivation. Psychology of Sport and Exercise, 4, 225-236.

Allegre, B., Therme, P. y Griffiths, M. (2007). Individual factors and the context of physical activity in exercise dependence: a prospective study of 'ultra-marathoners'. International Journal of Mental Health and Addiction, 5, 233-243.

Antunes, H., De Mello, M., de Aquino Lemos, V., Santos-Galduróz, R., Camargo Galdieri, L., Amodeo Bueno, O. y D’Almeida, V. (2015). Aerobic physical exercise improved the cognitive function of elderly males but did not modify their blood homocysteine levels. Dementia and Geriatric Cognitive Disorders Extra, 5(1), 13-24.

Antunes, H., Leite, G., Lee, K., Barreto, A., dos Santos, R., de Sá Souza, H. y De Mello, M. (2016). Exercise deprivation increases negative mood in exercise-addicted subjects and modifies their biochemical markers, Physiology Behavior, 156, 182-190.

Arbinaga, F. y Caracuel, J. C. (2007). Dependencia del ejercicio en fisicoculturistas competidores evaluada mediante la Escala de Adicción General Ramón y Cajal. Universitas Psychologica, 6, 549-558.

Bär, K. y Markser, V. (2013). Sport specificity of mental disorders: the issue of sport psychiatry. European Archives of Psychiatry and Clinical Neuroscience, 263, 205-210.

Becker, J., McClellan, M., y Reed, B. (2016). Sociocultural context for sex-differences in addiction. Addiction biology, 21, 1052-1059.

Berczik, K., Szabó, A., Griffiths, M., Kurimay, T., Kun, B., Urbán, R. y Demetrovics, Z. (2012). Exercise addiction: symptoms, diagnosis, epidemiology, and etiology. Substance Use and Misuse, 47, 403-417.

Berczik, K., Griffiths, M., Szabo, A., Kurimay, T., Kokonyei, G., Urbán, R. y Demetrovics, Z. (2014). Exercise addiction-the emergence of a new disorder. Australasian Epidemiologist, 27(2), 36.

Boecker, H., Sprenger, T., Spilker, M., Henriksen, G., Koppenhoefer, M., Wagner, K., Vale M., Berthele, A. y Tolle, T. (2008). The runner's high: opioidergic mechanisms in the human brain. Cerebral Cortex, 18, 2523-2531.

Buceta, J. M., López de la Llave, A., Pérez-Llantada, M., Vallejo, M. y Del Pino, M. (2003). Estado psicológico de los corredores populares de maratón en los días anteriores a la prueba. Psicothema, 15, 273-277.

Bueno, J., Capdevila, L. y Fernández-Castro, J. (2002). Sufrimiento competitivo y rendimiento en deportes de resistencia. Revista de Psicología del Deporte, 17, 209-226

Bueno, J., Weinberg, R., Fernandez-Castro, J. y Capdevila, L. (2008). Emotional and motivational mechanisms mediating the influence of goal setting on endurance athletes' performance. Psychology of Sport and Exercise, 9, 786-799.

Burtscher, J., Furtner, M., Sachse, P. y Burtscher, M. (2011). Validation of a German version of the Sport Motivation Scale (SMS28) and motivation analysis in competitive mountain runners. Perceptual and Motor Skills, 172, 807-820.

Cantón, E. (2016). La especialidad profesional en Psicología del Deporte. Revista de Psicología Aplicada al Deporte y al Ejercicio Físico, 1, e2. https://doi.org/10.5093/rpadef2016a2

Carmack, M. y Martens, R. (1979). Measuring commitment to running: A survey of runners' attitudes and mental states. Journal of Sport Psychology, 7, 25-42.

Carnes, P. (1983). The sexual addiction. Minneapolis: CompCare Publications.
Cernuda, V., de la Gándara Martín, J. y Alonso, M. (2015). Adicción al deporte: Estandarización de la Escala de Adicción al Deporte, SAS-15. Cuadernos de Medicina Psicosomática y Psiquiatría de Enlace, 116, 24-35.

Chapman, C. y De Castro, J. (1990). Running addiction: measurement. Journal Sports Medicine and Physical Fitness, 30, 283-90.

Cockerill, I. y Riddington, M. (1996). Exercise dependence and associated disorders: A review. Counselling Psychology Quarterly, 9, 119-129.

Cook, B., Karr, T. M., Zunker, C., Mitchell, J. E., Thompson, R., Sherman, R. y Wonderlich, S. A. (2013). Primary and secondary exercise dependence in a community-based sample of road race runners. Journal of Sport and Exercise Psychology, 35, 464-469.

Cousineau, D., Ferguson, R., De Champlain, J., Gauthier, P., Cote, P. y Bourassa, M. (1977). Catecholamines in coronary sinus during exercise in man before and after training. Journal of Applied Physiology, 43, 801-806.

Curtis, J. y McTeer, W. (1981). The motivation for running. Canadian runner, 1, 18-19.

Deci, E. y Ryan, R. (1985). The general causality orientations scale: Self-determination in personality. Journal of research in personality, 19(2), 109-134.

De Mello, M., de Aquino Lemos, V., Antunes, H., Bittencourt, L., Santos-Silva, R. y Tufik, S. (2013). Relationship between physical activity and depression and anxiety symptoms: a population study. Journal of Affective Disorders, 149, 241-246.

De la Vega, R., Rivera, O. y Ruíz, R. (2011). Personalidad Resistente en Carreras de Fondo: comparativa entre ultra fondo y diez kilómetros. Revista de Psicología del Deporte, 20, 445-454.

De La Vega, R., Parastatidou, I., Ruíz-Barquín, R., y Szabo, A. (2016). Exercise addiction in athletes and leisure exercisers: The moderating role of passion. Journal of Behavioral Addictions, 5(2), 325-331.

Dietrich, A. y McDaniel, W. (2004). Endocannabinoids and exercise. British Journal of Sports Medicine, 38, 536-541.

Doppelmayr, M. y Molkenthin, A. (2004). Motivation of participants in adventure ultramarathons compared to other foot races. Biology of Sport, 21, 319-323.

Dubreucq, S., Koehl, M., Abrous, D., Marsicano, G. y Chaouloff, F. (2010). CB1 receptor deficiency decreases wheel-running activity: consequences on emotional behaviours and hippocampal neurogenesis. Experimental Neurology, 224(1), 106-113.

Egorov, A. y Szabo, A. (2013). The exercise paradox: an interactional model for a clearer conceptualization of exercise addiction. Journal of Behavioral Addictions, 2(4), 199-208.

El Anuario de Estadísticas Deportivas (2016). Subdirección General de Estadística y Estudios Secretaría General Técnica. Ministerio de Educación, Cultura y Deporte.

Fairburn, C. G. (2008). Cognitive behavior therapy and eating disorders. London: Guilford Press.

Fernandez, M., Godoy-Izquierdo, D., Jaenes J. C., Bohorquez, M. y Velez, M. (2015). Flow y rendimiento en corredores de maratón. Revista de Psicología del Deporte, 24, 9-19.

Freimuth, M., Moniz, S. y Kim, S. (2011). Clarifying exercise addiction: differential diagnosis, co-occurring disorders, and phases of addiction. International Journal of Environmental Research and Public Health, 8(10), 4069-4081. 
Furst, D. y Germone, K. (1993). Negative addiction in male and female runners and exercisers. Perceptual and Motor Skills, 77(1), 192-194.

Gill, D. L. y Deeter, T. E. (1988). Development of the sport orientation questionnaire. Research Quarterly for Exercise and Sport, 59(3), 191-202

Glasser, W. (1976). Positive addiction. New York, Estados Unidos: Harper y Row.

Goldfarb, A. y Jamurtas, A. (1997). ß-Endorphin response to exercise. Sports Medicine, 24(1), 8-16.

González-Cutre, D., y Sicilia, Á. (2012). Motivation and exercise dependence: A study based on self-determination theory. Research Quarterly for Exercise and Sport, 83, 318-329.

Goodman, A. (1990). Addiction: definition and implications. British Journal of Addiction, 85, 1403-1408.

Grant, J., Potenza, M., Weinstein, A. y Gorelick, D. (2010). Introduction to behavioral addictions. The American Journal of Drug and Alcohol Abuse, 36, 233-241.

Griffiths, M. (1995). Adolescent gambling. London: Routledge.

Griffiths, M. (1996). Nicotine, tobacco and addiction. Nature, 384, 18.

Griffiths, M. (1997). Exercise addiction: A case study. Addiction Research, 5(2), 161-168.

Griffiths, M. (1999). Gambling technologies: Prospects for problem gambling. Journal of Gambling Studies, 15, 265-283.

Griffiths, M. (2000). Internet addiction: time to be taken seriously? Addiction Research, 8, 413-418.

Griffiths, M. y Blackwell. D. (2002). Gambling and gaming addictions in adolescence. Leicester: British Psychological Society.

Griffiths, M. (2005). A 'components' model of addiction within a biopsychosocial framework. Journal of Substance Use, 10(4), 191-197.

Griffiths, M., Szabo, A. y Terry, A. (2005). The exercise addiction inventory: a quick and easy screening tool for health practitioners. British Journal of Sports Medicine, 39(6), 30.

Griffiths, M., Urbán, R., Demetrovics, Z., Lichtenstein, M., de la Vega, R., Kun, B., Ruiz-Barquín, R., Yougman, J. y Szabo, A. (2015). A cross-cultural re-evaluation of the Exercise Addiction Inventory (EAI) in five countries. Sports Medicine-Open, 7(1), 5.

Hamer, M. y Karageorghis, C. (2007). Psychobiological mechanisms of exercise dependence. Sports Medicine, 37, 477-484

Hausenblas, H. y Downs, D. (2002). Exercise dependence: a systematic review. Psychology of Sport and Exercise, 3(2), 89-123.

Hausenblas, H. y Giacobbi, P. (2004). Relationship between exercise dependence symptoms and personality. Personality and Individual Differences, 36, 1265-1273.

Heazlewood, I., Walsh, J., Climstein, M., DeBeliso, M., Adams, K., Kettunen, J. y Munro, K. (2012). The motivations of marathoners scales instrument for evaluating motivational factors in a variety of mainstream sports. Journal of Science and Medicine in Sport, 15, Supplement 1, S137.

Heirene, R., Shearer, D., Roderique-Davies, G. y Mellalieu, S. (2016). Addiction in extreme sports: An exploration of withdrawal states in rock climbers. Journal of Behavioral Addictions, 5, 332-341.

Hollander, E. y Wong, C. M. (1995). Body dysmorphic disorder, pathological gambling, and sexual compulsions. Journal of Clinical Psychiatry, 56(4), 7-13.
Hulley, A. y Hill, A. (2001). Eating disorders and health in elite women distance runners. International Journal of Eating Disorders, 30, 312-317.

Jaenes, J. C. (2003). Entrenamiento psicológico para corredores de fondo y maratón. En S. Márquez (Coord.). Psicología de la Actividad Física y el Deporte: Una perspectiva Latina. (pp.343-348). León, España: Universidad de León.

Jaenes, J. C., Godoy, D. y Román, F. (2008). Elaboración y validación psicométrica de la escala de personalidad resistente en maratonianos (EPRM). Cuadernos de Psicología del Deporte, 8(2), 59-81.

Johnson, K. y Lovinger, D. (2016). Presynaptic G Protein-Coupled Receptors: Gatekeepers of Addiction? Frontiers in Cellular Neuroscience, 10, 264.

Johnston, O., Reilly, J. y Kremer, J. (2011). Excessive exercise: From quantitative categorisation to a qualitative continuum approach. European Eating Disorders Review, 19(3), 237-248.

Kanarek, R., D'anci, K., Jurdak, N. y Mathes, W. (2009). Running and addiction: precipitated withdrawal in a rat model of activity-based anorexia. Behavioral neuroscience, 123(4), 905.

Kerr, J., Lindner, K. y Blaydon, M. (2007). Exercise dependence. Nueva York, Estados Unidos: Routledge.

Konkolÿ, B., Woodin, E., Hodgins, D. y Williams, R. (2015). Natural course of behavioral addictions: a 5-year longitudinal study. BMC Psychiatry, 15(1), 4. http://dx.doi.org/10.1186/s12888015-0383-3

Kraemer, W., Fragala, M., van Henegouwen, W., Gordon, S., Bush, J., Volek, J., ... Mastro, A. (2013). Responses of proenkephalin Peptide $\mathrm{F}$ to aerobic exercise stress in the plasma and white blood cell biocompartments. Peptides, 42, 118-124.

Krouse, R., Ransdell, L., Lucas, S. y Pritchard, M. (2011). Motivation, goal orientation, coaching, and training habits of women ultrarunners. The Journal of Strength \& Conditioning Research, 25, 2835-2842.

Landolfi, E. (2013). Exercise addiction. Sports medicine, 43(2), 111-119.

Lane, A. y Wilson, M. (2011). Emotions and trait emotional intelligence among ultra-endurance runners. Journal of Science and Medicine in Sport, 14, 358-362.

Larumbe, E., Pérez-Llantada, M. y López de la Llave, A. (2009). Características del estado psicológico de los corredores populares de maratón. Revista de Psicología del Deporte, 18, 151-163.

Larumbe, E., Pérez-Llantada, M., López de la Llave, A. y Buceta, J. (2015). Desarrollo y características psicométricas preliminares del cuestionario PODIUM para corredores populares de maratón. Cuadernos de Psicología del Deporte, 15(3), 41-52.

Lendrum, J. (2015). Motivations, Limitations, and Guilt: Women who Marathon. Journal of Sporting Cultures and Identities, 6(3), 1-12.

Lichtenstein, M., Andries, A., Hansen, S., Frystyk, J. y Støving, R. (2015). Exercise addiction in men is associated with lower fat-adjusted leptin levels. Clinical Journal of Sport Medicine, 25(2), 138-143.

Lichtenstein, M., Larsen, K., Christiansen, E., Støving, R. y Bredahl, T. (2014). Exercise addiction in team sport and individual sport: prevalences and validation of the exercise addiction inventory. Addiction Research and Theory, 22, 437-437.

Macfarlane, L., Owens, G. y Cruz, B. (2016). Identifying the features of an exercise addiction: A Delphi study. Journal of Behavioral Addictions, 5, 474-484. 
Marlatt, G., Baer, J, Donovan, D., y Kivlahan, D. (1988). Addictive behaviors: Etiology and treatment. Annual Review of Psychology, 39, 223-252.

Márquez, S. y de la Vega, R. (2015). La adicción al ejercicio: un trastorno emergente de la conducta. Nutrición Hospitalaria, 31, 2384-2391.

Masters, K., y Lambert, M. (1989). The relations between cognitive coping strategies, reasons for running, injury, and performance of marathon runners. Journal of Sport and Exercise Psychology, 17(2), 161-170.

Masters, K., Ogles, B. y Jolton, J. (1993). The development of an instrument to measure motivation for marathon running: The Motivations of Marathoners Scales (MOMS). Research Quarterly for Exercise and Sport, 64, 134-143.

McNair, D. M., Lorr, M., y Droppleman, L. F. (1971). Profile of mood state manual. San Diego (CA), Estados Unidos: Educational and Industrial Testing Service.

McNamara, J. y McCabe, M. (2012). Striving for success or addiction? Exercise dependence among elite Australian athletes. Journal of Sports Sciences, 30, 755-766.

MacNicol, B. (2017). The biology of addiction. Canadian Journal of Anesthesia, 64(2), 141-148.

Meyer, C., Taranis, L., Goodwin, H. y Haycraft, E. (2011). Compulsive exercise and eating disorders. European Eating Disorders Review, 19(3), 174-189.

Modoio, V., Antunes, H., Gimenez, P., Santiago, M., Tufik, S. y Mello, M. (2011). Negative addiction to exercise: are there differences between genders? Clinics, 66, 255-260.

Morgan, W. (1979). Negative addiction in runners. Physician and Sports Medicine, 7, 57-70.

Orford, J. (2001). Excessive appetites: A psychological view of addictions. New York: John Wiley \& Sons Ltd.

Paradis, K., Cooke, L, Martin, L. y Hall, C. (2013). Too much of a good thing? Examining the relationship between passion for exercise and exercise dependence. Psychology of Sport and Exercise, 14, 493-500.

Parastatidou, I., Doganis, G., Theodorakis, Y. y Vlachopoulos, S. (2014). The mediating role of passion in the relationship of exercise motivational regulations with exercise dependence symptoms. International Journal of Mental Health and Addiction, 12, 406-419.

Pargman, D. (1980). The way of the runner: an examination of motives for running. En R.M. Suinn (Ed.), Psychology in Sports: Methods and Applications (pp. 90-98). Minneapolis, Estados Unidos: Burguess.

Pasman, L. y Thompson, J. (1988). Body image and eating disturbance in obligatory runners, obligatory weightlifters, and sedentary individuals. International Journal of Eating Disorders, 7 , $759-769$

Pinna, F., Dell'Osso, B., Di Nicola, M., Janiri, L., Altamura, A. C., Carpiniello, B. y Hollander, E. (2015). Behavioural addictions and the transition from DSM-IV-TR to DSM-5. Journal of Psychopathology, 21, 380-389.

Pugh, N. y Hadjistavropoulos, H. (2011). Is anxiety about health associated with desire to exercise, physical activity, and exercise dependence? Personality and Individual Differences, 51, 1059-1062.

Robbins, J., y Joseph, P. (1985). Experiencing exercise withdrawal: Possible consequences of therapeutic and mastery running. Journal of Sport Psychology, 7(1), 23-39.
Rudy, E. y Estok, P. (1990). Running addiction and dyadic adjustment. Research in nursing \& health, 13, 219-225.

Ruiz-Juan, F. y Zarauz, A. (2011). Validación de la versión española de la Commitment to Running Scale (CR). Estudios de Psicología, 32(2), 195-207

Ruiz-Juan, F. y Zarauz, A. (2012). Variables que hacen adicto negativamente a correr al maratoniano español. Retos, 21, 38-42.

Ruiz-Juan, F., Sancho, A. Z. e Ibarzábal, F. A. (2013). Validación de la Escala de Adicción al Entrenamiento (EAE) en atletas veteranos. Adicciones, 25, 309-320.

Ruiz-Juan, F. y Zarauz, A. (2016). Predictores de la adicción en atletas veteranos españoles. Suma Psicológica, 23(1), 10-17.

Ruiz-Juan, F., Zarauz, A. y Flores-Allende, G. (2016a). Precompetitive anxiety in long-distance runners depending on their training variables. Retos-Nuevas Tendencias en Educación Física, Deporte y Recreación, 30, 110-113.

Ruiz-Juan, F., Zarauz, A. y Flores-Allende, G. (2016b). Dependencia al entrenamiento y competición en corredores de fondo en ruta. CCD. Cultura, Ciencia, Deporte. 17(32), 149-155.

Rusbult, C., Martz, J. y Agnew, C. (1998) The Investment Model Scale: measuring commitment level, satisfaction level, quality of alternatives, and investment size. Personal Relationships, 5, 357-391.

Sachs, M. L. (1981). Running addiction. En: Sachs, M. H and Sacks, M. L. (Eds.), Psychology of running (pp.116-126). Champaign, IL, Estados Unidos: Human Kinetics.

Sancho, A. y Ruiz-Juan, F. (2011). Psychometric properties of the Spanish version of the Running Addiction Scale (RAS). The Spanish Journal of Psychology, 14, 967-976.

Sanz, T., Blasco, T. y Cruz, J. (1992). Adicción a la actividad física de fondo. Archivos de Medicina del Deporte, 9, 279-286.

Scanlan, T., Carpenter, P., Simons, J., Schmidt, G. y Keeler, B. (1993). An introduction to the sport commitment model. Journal of Sport and Exercise Psychology, 15(1), 1-15.

Salazar, J., Castro, S., Remicio, C., Zambrano, S. y Colombia, S. (2012). Niveles de adicción al ejercicio corporal en personas fisioculturistas. Revista Iberoamericana de Psicología del Ejercicio y el Deporte, 7, 209-226.

Shipway, R. y Holloway, I. (2010). Running free: Embracing a healthy lifestyle through distance running. Perspectives in Public Health, 130(6), 270-276.

Slay, H., Hayaki, J., Napolitano, M. y Brownell, K. (1998). Motivations for running and eating attitudes in obligatory versus nonobligatory runners. International Journal of Eating Disorders, 23, 267-275.

Smith, D., Wright, C. y Winrow, D. (2010). Exercise dependence and social physique anxiety in competitive and non competitive runners. International Journal of Sport and Exercise Psychology, 8(1), 61-69.

Stoll, O. (1997). Endorphine, Laufsucht und runner's high. Aufstieg und niedergang eines mythos [Endogenous opiates, "runner's high" and "Exercise Addiction" - the rise and decline of a myth]. Leipziger Sportwissenschaftliche Beitraege, 28(1), 102-121.

Suckling, J. y Nestor, L. (2017). The neurobiology of addiction: the perspective from magnetic resonance imaging present and future. Addiction, 112, 360-369.

Summers, J., Machin, V. y Sargent, G. (1983) Psychosocial factors related to marathon running. Journal of Sport Psychology, $5,314-331$ 
Summers, J., Sargent, G., Levey, A. y Murray, K. (1982). Middle-aged, non-elite marathon runners: A profile. Perceptual and Motor Skills, 54, 963-969.

Summers, J. S. (2015). What is wrong with addiction. Philosophy, Psychiatry, and Psychology, 22(1), 25-40.

Sussman, S., Leventhal, A., Bluthenthal, R., Freimuth, M., Forster, M. y Ames, S. (2011). A framework for the specificity of addictions. International Journal of Environmental Research and Public Health, 8, 3399-3415.

Szabo, A. (1995). The impact of exercise deprivation on well-being of habitual exercisers. The Australian Journal of Science and Medicine in Sport, 27, 68-75.

Szabo, A. (2010). Addiction to exercise: A symptom or a disorder? New York, Estados Unidos: Nova Science.

Szabo, A., Frenkl, R. y Caputo, A. (1997). Relationships between addiction to running, commitment to running and deprivation from running: A study on the internet. European Yearbook of Sport Psychology, 1, 130-147.

Szabo, A. y Griffiths, M. D. (2007). Exercise addiction in British sport science students. International Journal of Mental Health and Addiction, 5(1), 25-28.

Szabo, A., Griffiths, M. D. y Demetrovics, Z. (2016) Exercise Addiction. En Preedy, V. R. (Ed.). Neuropathology of Drug Addictions and Substance Misuse Volume 3: General Processes and Mechanisms, Prescription Medications, Caffeine and Areca, Polydrug Misuse, Emerging Addictions and Non-Drug Addictions (pp. 984992). London, Reino Unido: Academic Press.

Szabo, A., De La Vega, R., Ruiz-Barquín, R. y Rivera, O. (2013). Exercise addiction in Spanish athletes: Investigation of the roles of gender, social context and level of involvement. Journal of behavioral addictions, 2, 249-252.

Terry, A., Szabo, A. y Griffiths, M. (2004). The Exercise Addiction Inventory: a new brief screening tool. Addiction Research and Theory, 12, 489-499.

Thompson, J. y Blanton, P. (1987). Energy conservation and exercise dependence: a sympathetic arousal hypothesis. Medicine and Science in Sports and Exercise, 19, 91-97.

Thompson, J, y Pasman, L. (1991). The obligatory exercise questionnaire. The Behavior Therapist, 14, 137.

Thornton, E. y Scott, S. (1995). Motivation in the committed runner: Correlations between self-report scales and behaviour. Health Promotion International, 10(3), 177-184.

Veale, D. (1987). Exercise dependence. Addiction, 82, 735-740.

Veale, D. (1995). Does Primary Exercise Dependence really exist? En J. Annett, B. Cripps, y H.

Steinberg (Eds.), Exercise Addiction: Motivation for participation in sport and exercise: Proceedings of British Psychology, Sport and Exercise Psychology Section (pp. 71-75). British Psychological Society.
Vealey, R. S. (1986). Conceptualization of sport-confidence and competitive orientation: Preliminary investigation and instrument development. Journal of sport psychology, 8, 221-246.

Warburton, D.., Nicol, C. y Bredin, S. (2006). Health benefits of physical activity: the evidence. Canadian Medical Association Journal, 174, 801-809.

Weinstein, A. y Weinstein, Y. (2014). Exercise addiction-diagnosis, bio-psychological mechanisms and treatment issues. Current Pharmaceutical Design, 20, 4062-4069.

Wichmann, S. y Martin, D. (1992). Exercise excess: treating patients addicted to fitness. Physician and Sports Medicine, 20(5), 193-200.

Yates, A. (1991). Compulsive exercise and the eating disorders: Toward an integrated theory of activity. New York, Estados Unidos: Brunner-Mazel.

Youngman, J. y Simpson, D. (2014). Risk for exercise addiction: A comparison of triathletes training for sprint, Olympic, half-ironman and ironman-distance triathlons. Journal of Clinical Sport Psychology, 8(1), 19-37.

Zach, S., Xia, Y., Zeev, A., Arnon, M., Choresh, N. y Tenenbaum, G. (2015). Motivation dimensions for running a marathon: A new model emerging from the Motivation of Marathon Scale (MOMS). Journal of Sport and Health Science. http://dx.doi.org/10.1016/j.jshs.2015.10.003

Zarauz, A. y Ruiz-Juan, F., (2011). Validación al español de las escalas motivaciones de los maratonianos (MOMS), compromiso a correr (CR) y adicción a correr (RAS). Variables predictoras de la "súper-adherencia" y las motivaciones de los maratonianos. (Tesis doctoral). Universidad de Murcia. Murcia.

Zarauz, A., y Ruiz-Juan, F., (2012). Super-Adherence of Marathoners: Predictor Variables and Gender Differences. Universitas Psychologica, 11, 895-907.

Zarauz, A. y Ruiz-Juan, F., (2014). Factores determinantes de la ansiedad en atletas veteranos españoles. Universitas Psychologica, 13, 1047-1058.

Zarauz, A., Ruiz-Juan, F., Arbinaga, F., Jaenes, J. C., y Flores-Allende, G. (2015). Modelo predictor de las motivaciones para correr: análisis con corredores de ruta españoles vs. mexicanos. Universitas Psychologica, 14, 659-674.

Zarauz, A., Ruiz-Juan, F. y Flóres-Allende, G. (2016). Modelos predictores de la motivación en corredores de fondo en ruta en función de sus hábitos de entrenamiento. Revista Iberoamericana de Psicología del Ejercicio y el Deporte, 17, 185-192.

Zingg, M., Knechtle, B., Rüst, C., Rosemann, T. y Lepers, R. (2013). Reduced performance difference between sexes in master mountain and city marathon running. Interantioanl Journal of General Medicine, 6, 267-275. 